Répertoire des céramiques de Rennes aux XVe et XVIe siècles, à partir des découvertes du site hospitalier de la place Sainte-Anne (Ille-et-Vilaine, Bretagne)

Pottery of Rennes in the 15th and 16th centuries, from the discoveries of the hospital site of Sainte-Anne square (Ille-et-Vilaine, Bretagne)

Françoise Labaune-Jean et Dominique Pouille (avec la collaboration de)

\title{
OpenEdition
}

Journals

Édition électronique

URL : https://journals.openedition.org/rao/1793

DOI : 10.4000/rao.1793

ISBN : 978-2-7535-2790-4

ISSN : 1775-3732

Éditeur

Presses universitaires de Rennes

Édition imprimée

Date de publication : 30 décembre 2012

Pagination : 259-286

ISBN : 978-2-7535-2641-9

ISSN : 0767-709X

Référence électronique

Françoise Labaune-Jean et Dominique Pouille (avec la collaboration de), «Répertoire des céramiques de Rennes aux XVe et XVle siècles, à partir des découvertes du site hospitalier de la place Sainte-Anne (Ille-et-Vilaine, Bretagne) », Revue archéologique de l'Ouest [En ligne], 29 | 2012, mis en ligne le 30 décembre 2014, consulté le 22 juillet 2021. URL : http://journals.openedition.org/rao/1793 ; DOI : https://doi.org/10.4000/rao.1793

Ce document a été généré automatiquement le 22 juillet 2021.

@ Presses universitaires de Rennes 


\section{Répertoire des céramiques de Rennes aux XVe et XVIe siècles, à partir des découvertes du site hospitalier de la place Sainte-Anne (Ille-et-Vilaine, Bretagne)}

Pottery of Rennes in the 15th and 16th centuries, from the discoveries of the hospital site of Sainte-Anne square (Ille-et-Vilaine, Bretagne)

Françoise Labaune-Jean et Dominique Pouille (avec la collaboration de)

\section{NOTE DE L'ÉDITEUR}

Manuscrit reçu le 27 juin 2011, accepté le 10 novembre 2011.

Dans le cadre de la mise en place de la première ligne de métro-Val de Rennes, la fouille de la station "place Sainte-Anne " s'est déroulée de janvier à juin 1998 (Pouille, 1998-2000; fig. 1). En 1992, les sondages d'évaluation réalisés avaient permis de détecter la présence de vestiges antiques et médiévaux à cet emplacement. Ces derniers, très mal conservés en raison de l'écrêtement de la stratigraphie, semblaient cependant n'être qu'anecdotiques. Seules les études d'archives réalisées dans le cadre d'une recherche documentaire préalable permettaient de percevoir la nature exacte de l'occupation des lieux aux époques médiévale et moderne (Goupil et Cozic, 1991). 
Figure 1 : Positionnement des vestiges et de l'emprise de la station de métro sur une restitution de la topographie du quartier dans sa configuration des années 1500-1550. D'après l'étude d'archives de N. Cozic ; innfographie : M. Dupré et F. Labaune-Jean, Inrap.

Figure 1: Location of vestiges and influence of the subway station on the topography of the district in its configuration of the years 1500-1550. According to the study of archives of N. Cozic.

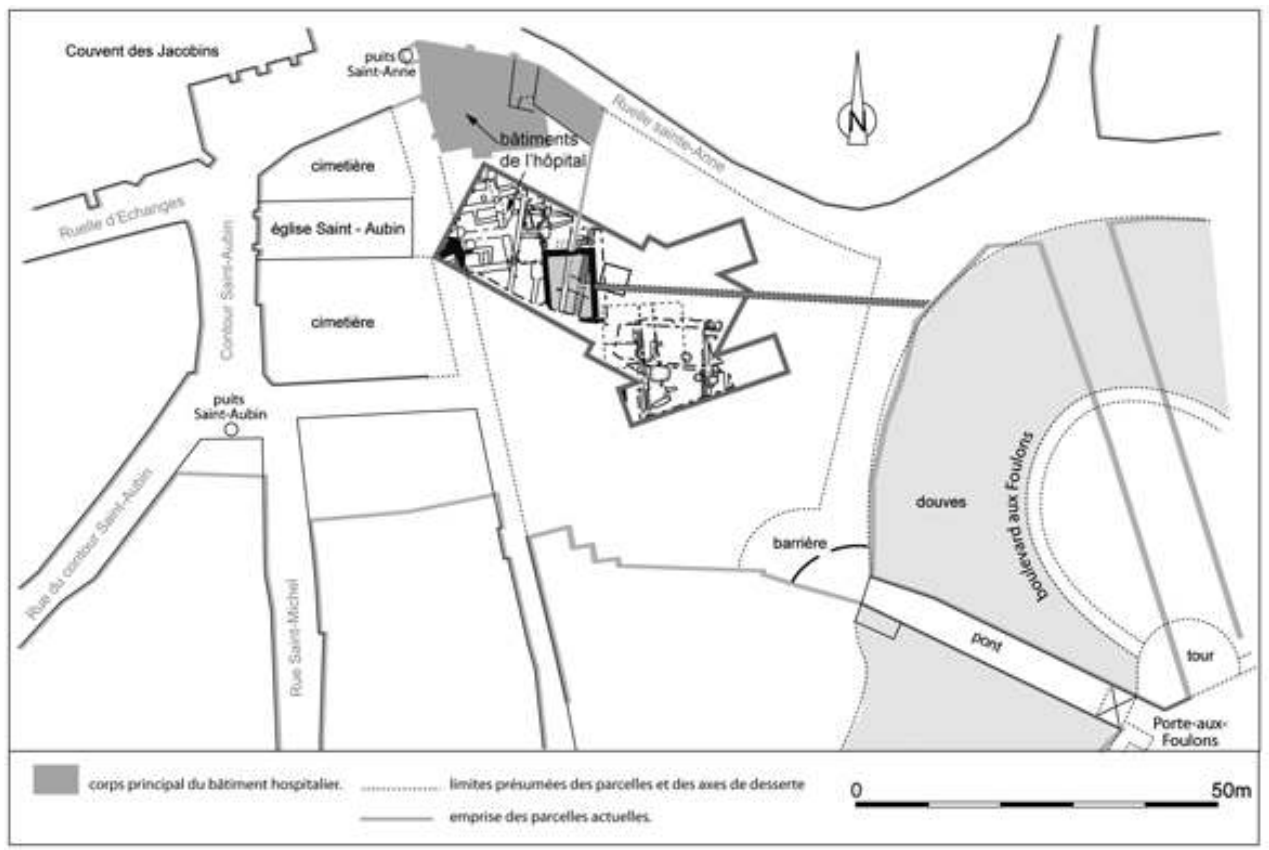

2 Situé « hors les murs », le secteur de l'actuelle place Sainte-Anne est localisé à moins de 200 mètres au nord de la cité de Rennes: seule partie de la ville à être dotée de murailles depuis l'Antiquité. Suite à l'édification, de 1421 à 1448, de la seconde enceinte de Rennes (Dubant, 2003, p. 50), ce faubourg n'est plus qu'à quelques dizaines de mètres de la barbacane la plus septentrionale du secteur nouvellement fortifié. C'est dans ce suburbium, dont l'urbanisation médiévale remonte au moins au XII siècle, qu'a été fondé en 1340 l'hôpital Sainte-Anne. L'église Saint-Aubin, voisine de l'hôpital, est mentionnée dès 1158 comme faisant partie des faubourgs de la cité (ecclesia sancti albini in suburbio constitua) (Arch. Dép. 35, 1F501/1, copie du cartulaire de Saint-Mélaine, fo32, acte $\left.n^{\circ} 22\right)$. Cette ouverture d'une structure hospitalière nouvelle, menée sous l'impulsion de dix confréries ouvrières de Rennes, est une réponse à l'essor urbain que connaît la capitale bretonne en ce début de XIV siècle (Rennes, arch. mun., Acte de fondation de l'hôpital Sainte-Anne, Décembre 1340, Liasse 327). En effet, « élan urbain et élan hospitalier sont intimement liés "(Le Goff, 1980, p. 349). C'est également l'époque des crises démographiques provoquées par les épidémies et les famines précédant la Peste Noire, qui touchera la Bretagne en 1348.

\section{Le contexte}

Cet hôpital, l'un des six ou sept équipant la ville, comprenait principalement une salle des malades et une chapelle. D'autres bâtiments, de moindre importance, situés à l'intérieur d'un enclos, complétaient cet ensemble. C'est une partie des vestiges de ces derniers qui fut appréhendée lors des fouilles de la station de métro. Si les ensembles céramiques mis au jour sur l'emprise de la station de métro couvrent une période allant du début $d u X V^{e}$ à la première moitié $d u X V I I^{e}$ siècle, cette présentation se limite aux 
deux contextes les plus importants quantitativement et appartenant chronologiquement à la période deuxième moitié $d u X V^{e}$-première moitié $d u X I^{\mathrm{e}}$ siècle, période pour laquelle l'étude est jusqu'alors inédite sur Rennes : les latrines aménagées (L1) et la vaste cuve d'une buanderie utilisée comme dépotoir avant son remblaiement définitif au milieu du XVI ${ }^{\mathrm{e}}$ siècle (D2).

Les contraintes imposées lors de la réalisation des sondages n'avaient pas permis d'obtenir une vision satisfaisante de l'état de conservation des vestiges sur l'ensemble du site (fig. 2). Dès le début du décapage archéologique, il s'est avéré qu'une bonne partie de l'emprise de la fouille était occupée par un immense creusement correspondant probablement à une carrière médiévale d'extraction de pierres. Aucune mention n'en a été retrouvée dans les archives, cependant d'autres exemples sont mentionnés dans plusieurs secteurs de la ville (Cozic, 1998-2000, p. 14-15). De plus, un écrêtement général du site avait fait disparaître la plupart des vestiges postérieurs, ne conservant que les structures excavées.

Figure 2 : Plan général des structures fouillées, avec localisation de la buanderie-dépotoir D2 et de la fosse-latrines L1. Infographie : M. Dupré et F. Labaune-Jean, Inrap.

Figure 2: General plan of the structures, with localization of the laundry-garbage dump D2 and the pit-latrines L1.

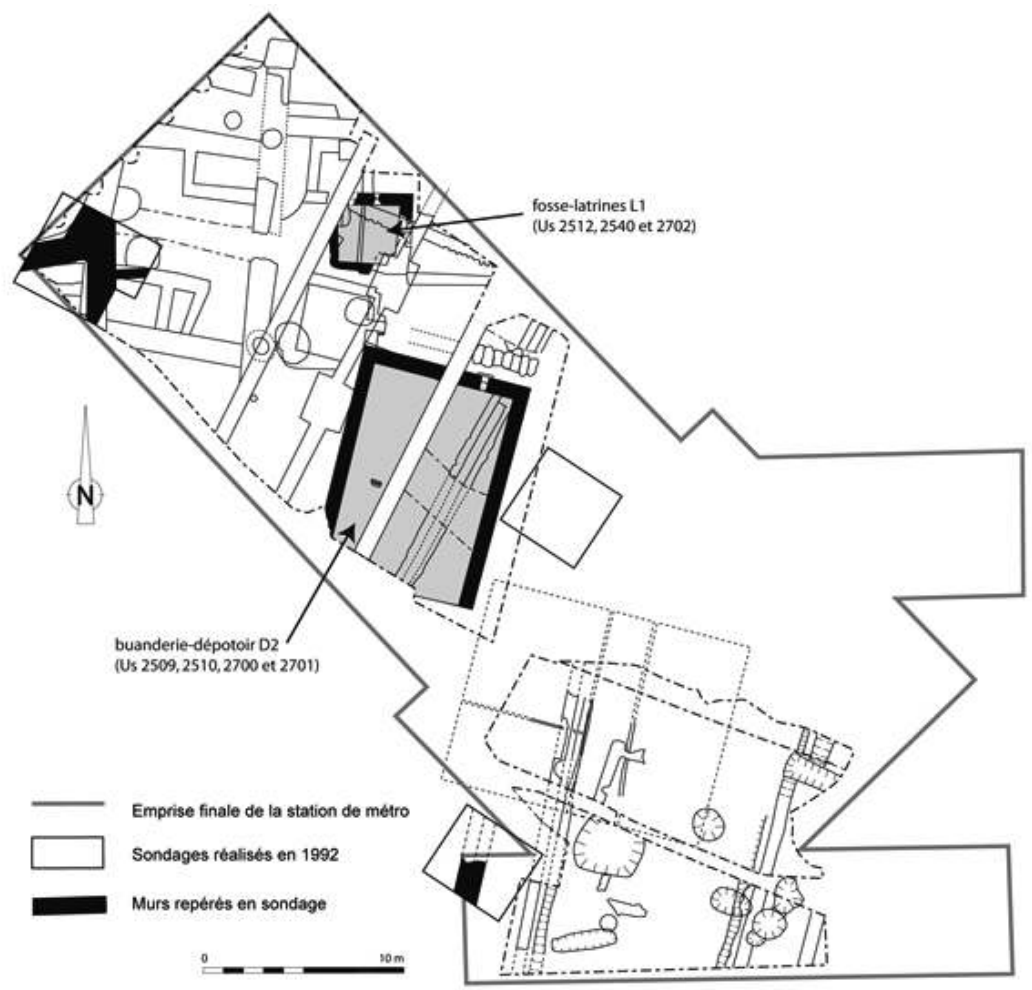

Le premier ensemble retenu, les latrines ( $\mathrm{L} 1$ = Us 2512, 2540 et 2702), se compose d'une maçonnerie de forme carrée en blocs de schiste, délimitant un petit espace pourvu d'un escalier. Cet aménagement se place en bordure de la carrière médiévale. Au cours de leur utilisation, elles ont fait l'objet de plusieurs remaniements, telle l'installation de sièges le long du mur, avant d'être condamnées progressivement par l'aménagement des espaces extérieurs. Pour cette raison, le mobilier se répartit en trois couches stratigraphiques. Faute d'études précises sur ce type de mobilier, il est actuellement difficile d'établir une chronologie relative au sein de ces ensembles de la seconde 
moitié $\mathrm{du} \mathrm{Xv}^{\mathrm{e}}$ siècle. Le mobilier mis au jour comprend essentiellement des récipients en céramique constituant un vaisselier riche en formes, complété par quelques verres. Le tout correspond au remblaiement d'une structure dans la première moitié du $\mathrm{xvI}^{\mathrm{e}}$ siècle, mais avec du mobilier de la seconde moitié $\mathrm{du} \mathrm{Xv}^{\mathrm{e}}$ siècle, datation confirmée par une monnaie frappée entre 1500 et 1550 (Pilet-Lemière, 1998-2000).

Dans son état d'origine, dont la construction est située à la charnière des $\mathrm{XV}^{\mathrm{e}}$ $\mathrm{XVI}^{\mathrm{e}}$ siècles, la buanderie (D2) réutilisait pour partie le creusement de la rampe d'accès à la carrière. Des maçonneries aménagées à l'intérieur de ce dernier constituaient un espace servant de réserve d'eau. Les dimensions intérieures de la buanderie sont de 7 mètres par 10,70 mètres. La profondeur de la cuve, jusqu'au niveau du trop-plein est de 5 mètres avec un surcreusement de près de 2 mètres en partie centrale. Au total, on obtient une contenance d'au moins 400 mètres cubes. Elle devait être alimentée par le nord, sans doute, en partie par les toitures de la structure hospitalière. Sur la face est de l'édifice, était prévu un système de trop-plein devant initialement être relié par une canalisation aux douves de la ville intra-muros situées non loin de là (fig. 3). La fouille a montré que la buanderie est restée inachevée pour une raison encore inconnue ${ }^{1}$. Elle n'a jamais été dotée de sa canalisation d'alimentation en eau et de son canal de tropplein.

Figure 3 : Vue de la cuve de la buanderie en cours de fouille. Cliché. D. Pouille, Inrap. Figure 3: View of the tank of the laundry in the course of search.

7 Rapidement, soit une estimation de moins d'une quarantaine d'années, la cuve a été transformée en dépotoir, fournissant ainsi à la fouille une extraordinaire quantité de matériel archéologique. Outre les classiques céramiques, on compte bon nombre de verres, des petits objets de la vie quotidienne: couteaux, enseignes de pèlerins, médailles, monnaies, épingles, perles, jeux de marelle ou dessins de bateaux gravés sur des ardoises ou des carreaux de terre-cuite... Avec un terminus situé en 1553 sous le règne d'Henri II, le petit lot monétaire ( 25 monnaies et jetons) de faible valeur ( 5 sous 6 deniers) reflète bien la circulation monétaire dans les milieux populaires au milieu du $\mathrm{XVI}^{\mathrm{e}}$ siècle. (Pilet-Lemière, 1998-2000). Ce milieu présentait également la particularité d'être toujours en eau, avec des comblements hermétiques riches en détritus végétaux, permettant ainsi la conservation de nombreuses pièces organiques : écuelles, peignes, cuillères et objets divers en bois, quelques milliers de fragments de cuirs (chaussures, sacoches), quelques fragments d'étoffes, d'innombrables graines et noyaux divers, des insectes et une grande variété de parasites. De nombreux ossements animaux issus d'équarrissage mais aussi d'origine alimentaire complètent cette liste. L'ensemble présente un profil très homogène datable de la première moitié du XvI ${ }^{e}$ siècle.

\section{2. Étude typologique du mobilier céramique}

8 Pour simplifier la présentation et en l'absence de différences dans le faciès céramique, les couches stratigraphiques sont réunies par contexte. Les numéros de structure d'origine sont remplacés par des noms génériques : latrines L1 (us 2512, 2540 et 2702) et dépotoir D2 (Us 2509, 2510, 2700 et 2701). Les différentes formes recueillies sont décrites séparément, selon les grands groupes et les critères de classification établis par I-ceramm ${ }^{2}$. 
Sur un total de 85978 tessons avant remontage, les individus ont été dénombrés à partir des bords différents auxquels s'ajoutent des éléments morphologiques particuliers. (fig.4). À lui seul, le dépotoir principal D2 comprend des quantités impressionnantes de mobilier et un bon état de conservation, soit près de 72000 tessons (8315 NMI) de céramique dont une cinquantaine de vases intacts, une centaine d'autres complets pour un poids total supérieur à deux tonnes, qui s'expliquent par les dimensions importantes de l'aménagement du dépotoir D2. Pour les latrines L1, on dispose d'un ensemble d'un nombre de restes de 14014 pour 714 NMI.

Figure 4 : Aperçu de quelques-unes des formes recueillies dans le comblement de la buanderie. Cliché H. Paitier, Inrap.

Figure 4: Some of the forms collected in the filling of the laundry.

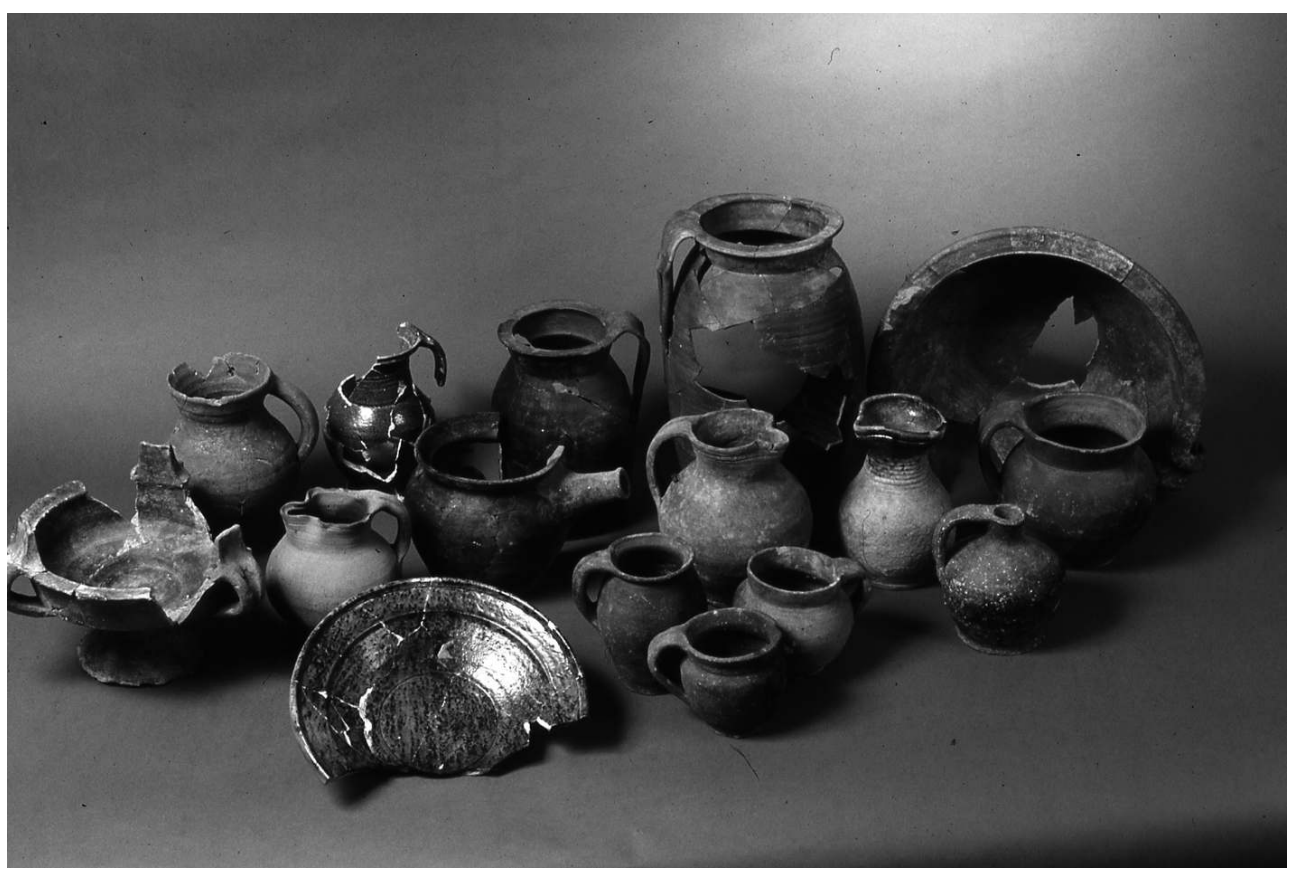

\section{Les groupes techniques}

Un premier examen visuel des récipients a permis de distinguer différents groupes en fonction de l'aspect de la pâte et des revêtements. Ce classement n'a pas encore été complété par une analyse physico-chimique ou pétrographique. La totalité du mobilier recueilli peut être répartie selon huit groupes principaux. Ici, nous renvoyons le lecteur au site internet d'I-ceramm, pour consulter les clichés des tranches et les états de surface de ces groupes techniques référencés sous le même encodage, complété par l'abréviation « ren » pour identifier les lots du site de l'hôpital Sainte-Anne.

\section{Groupe 1 (GT1)}

11 La pâte est presque toujours bicolore en tranche (orange à franges jaune ou grise à franges brun à orange). Les inclusions visibles renferment essentiellement des grains de quartz de taille variable suivant les formes $(0,05$ à $0,2 \mathrm{~cm})$. Quelques exemplaires contiennent de la chamotte. La surface présente toutes les nuances du brun clair au gris 
foncé. Les tessons placés dans ce lot ne portent aucun revêtement. Compte tenu de la présence de nombreux ratés de cuisson au sein du lot D2 (cf. infra), la distinction cuisson oxydante/réductrice n'a pas pu être réalisée de façon certaine. Une partie du lot est très cuite à la limite du grésage en surface.

Cet ensemble correspond au groupe générique de la céramique de production locale. Il renferme plus de $90 \%$ des tessons (sur le total des deux lots) et la majorité des formes produites (22 sur 46 types distincts, si l'on comptabilise uniquement les grands types, sans leurs variantes). Cette importance quantitative reflète une provenance locale, attestée par les archives, avec un approvisionnement fourni par les ateliers de potiers de Chartres-de-Bretagne (Ille-et-Vilaine). L'examen visuel des pâtes confirme cette attribution par comparaison avec les tessons issus de la fouille des ateliers. Même s'il s'agit des formes produites par les ateliers chartrains entre le $\mathrm{x}^{\mathrm{e}}$ et le xiII ${ }^{\mathrm{e}}$ siècle, la composition des pâtes présente de fortes similitudes. (Beuchet et al., 1990 ; fig. 5).

Figure 5 : Vue d'un coquemar (pot 3) à rattacher aux productions chartraines. Cliché H. Paitier, Inrap.

Figure 5: View of a coquemar (jar 3) to connect with the productions of Chartres-de-Bretagne.

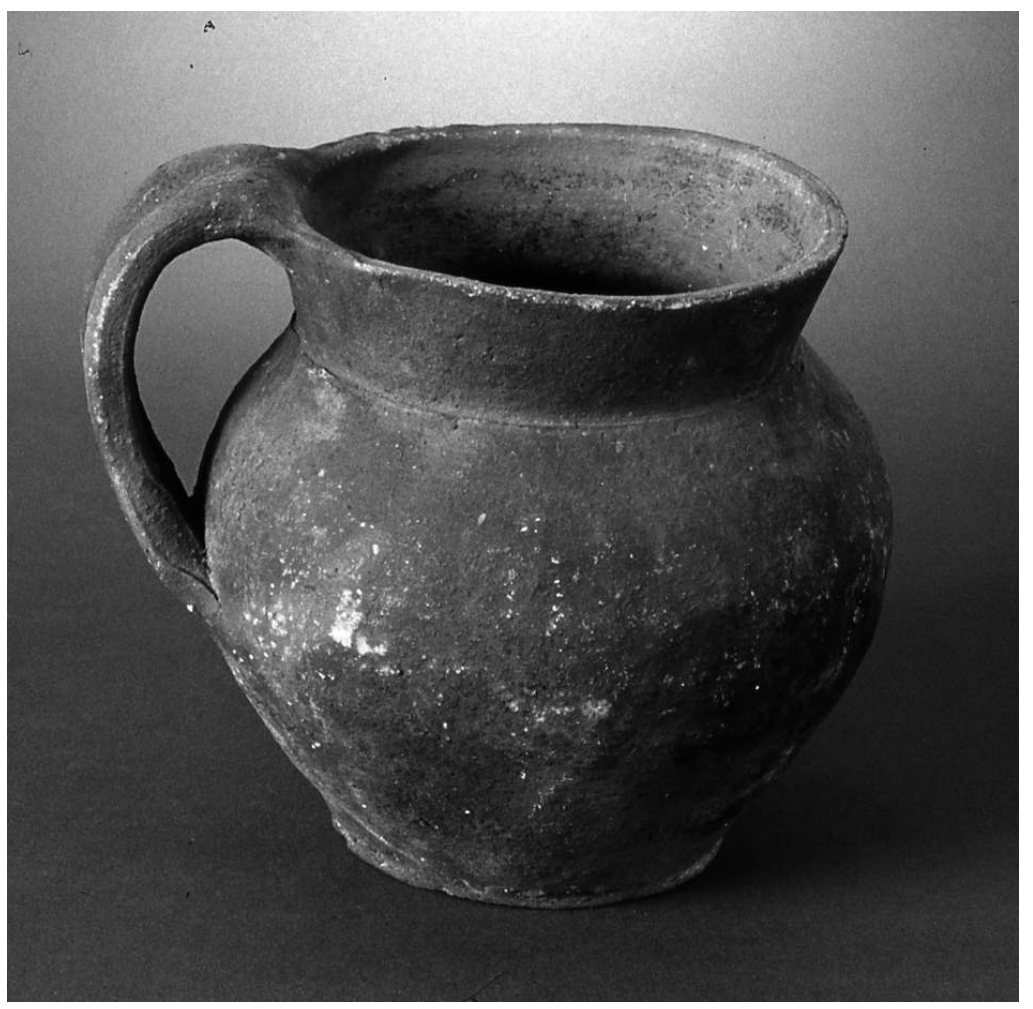

Seul un petit lot reste à isoler au sein de ce groupe (GT1-2). Il regroupe des tessons à pâte noire, renfermant des inclusions très denses et de granulométrie moyenne (1 à $2 \mathrm{~mm}$ ). Ces dernières donnent un aspect granuleux à la surface lissée brute. Une étude complémentaire est nécessaire ici pour en déterminer la provenance et voir s'il peut s'agir d'une production spécifique du groupe de Chartres de Bretagne car, par manque de temps pendant la post-fouille, le comptage de ce groupe n'a pas été terminé et demanderait à être repris. Il est signalé ici pour être mis en association à quelques formes typologiques particulières (fig. 6). 
Figure 6 : Vue d'un coquemar (pot 3) à pâte granuleuse. Cliché H. Paitier, Inrap. Figure 6: View of a coquemar (jar 3) with granular fabric.

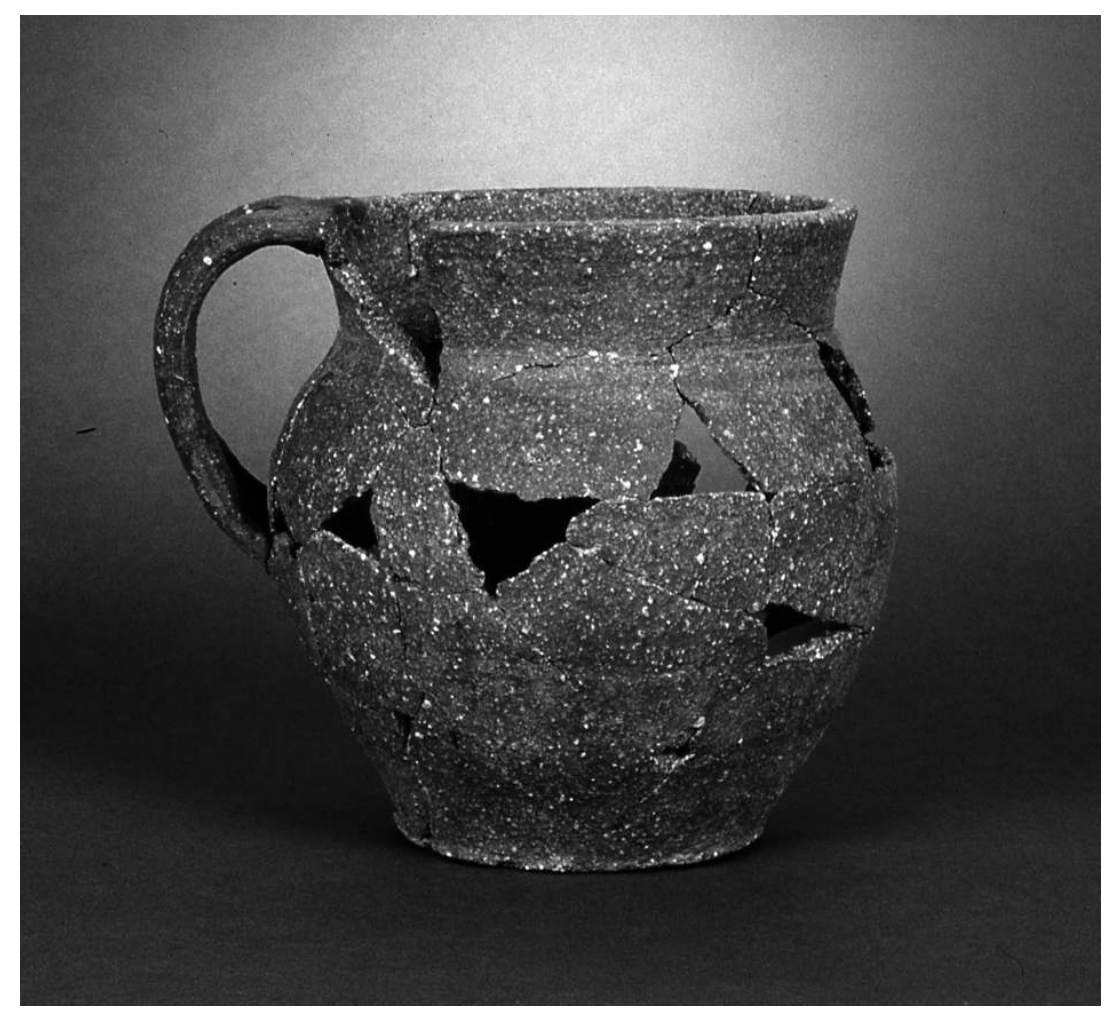

\section{Groupe 2 (GT2)}

14 La pâte est toujours à cuisson oxydante. La teinte couvre les dégradés de jaune pâle à orange clair. Elle est associée à un revêtement glaçuré plus ou moins couvrant, apposé sur la face externe ou interne. Des distinctions interviennent suivant les combinaisons des teintes de la pâte et de la glaçure (fig. 7).

Figure 7 : Aperçu de quelques-unes des formes glaçurées recueillies. Cliché H. Paitier, Inrap. Figure 7: Perceived of some of the forms with glaze.

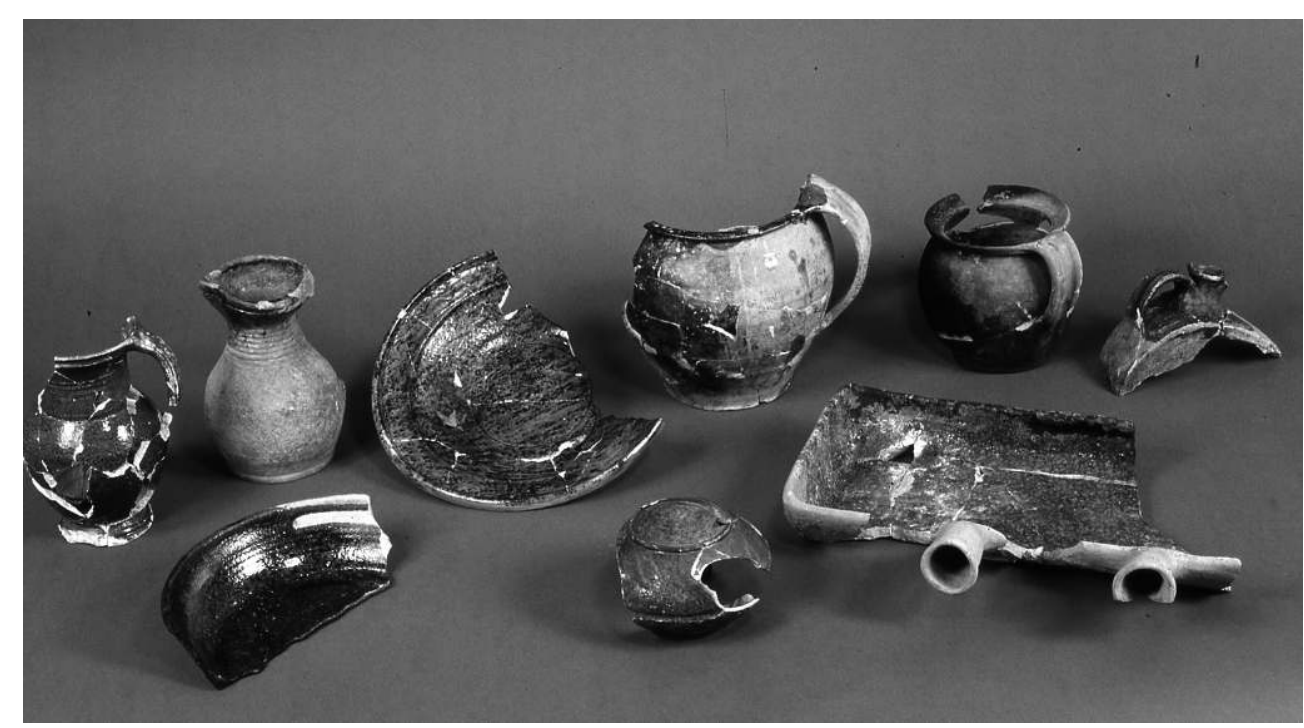



vert sombre. Cette production majoritaire (soit 14 des 46 types de formes) est à attribuer aux ateliers de Chartres-de-Bretagne, par comparaison avec les tessons issus des rebuts. nombreuses nuances de coloris. Cette production semble correspondre aux céramiques glaçurées de Saintonge (Chapelot, 1975-1976).

Groupe 2-3: pâte très claire blanche à beige avec glaçure plombifère uniforme et couvrante, de teinte vert moyen, d'aspect granuleuse. Malgré cet aspect facilement identifiable, la provenance de ces récipients reste indéterminée pour le moment.

Groupe 2-4 : pâte clair blanche à beige, associée à une glaçure externe vert clair, dense, appliquée avec soin sur la surface externe des récipients. Ces caractéristiques de pâte associées à des formes spécifiques permettent de les comparer aux productions des ateliers du Beauvaisis (fig. 8).

Figure 8 : Vue d'un pichet glaçuré du groupe technique 2-4. Cliché H. Paitier, Inrap. Figure 8: View of a glazed jug of the technical group 2-4.

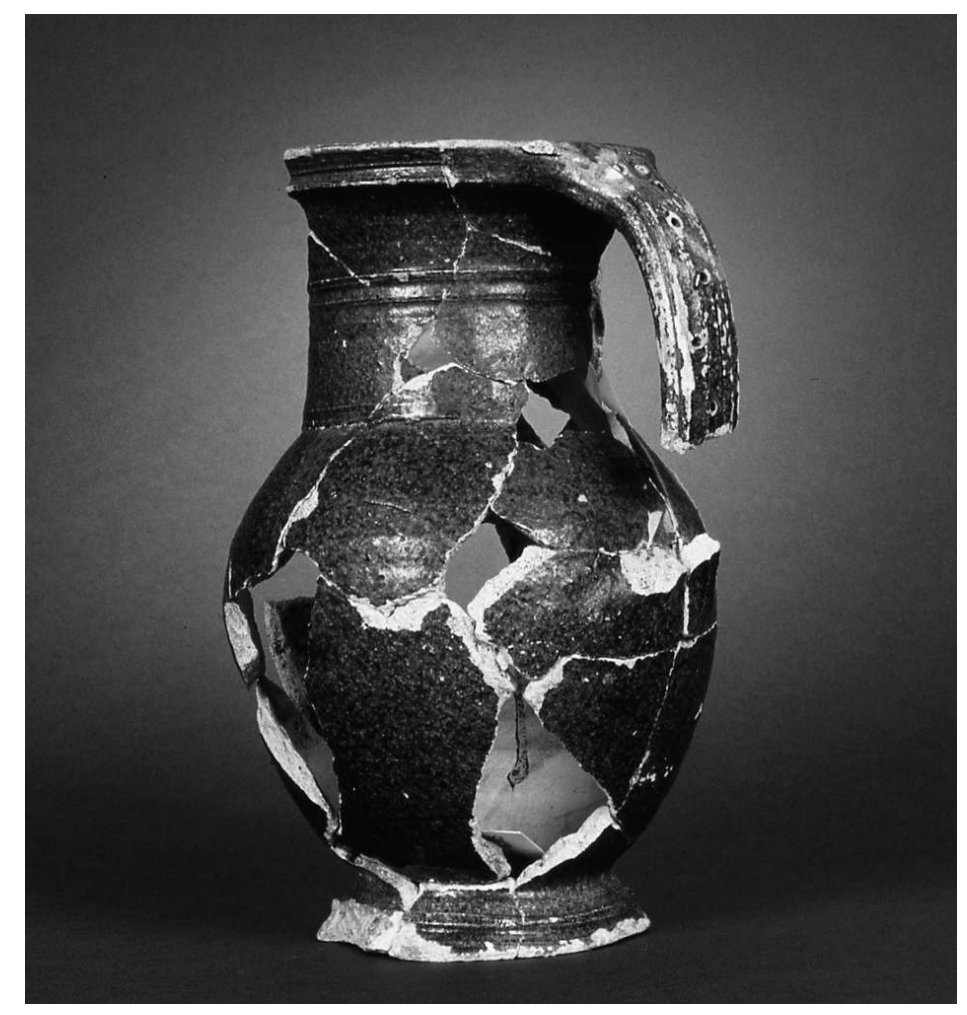

\section{Groupe 3 (GT3)}

Bien qu'appartenant au groupe de la céramique de production locale sans revêtement (GT1), ce lot est individualisé en raison de sa pâte spécifique permettant de distinguer une production particulière: il s'agit de la céramique dite "rose bleue» de Laval. La pâte est facilement identifiable grâce à une face interne de teinte gris bleu et la face externe orange à brun clair. Ces couleurs se trouvent en surface, aucun revêtement ne venant les recouvrir. Les inclusions de feldspath donnent un aspect granuleux à la 
surface. La pâte est très cuite et sonore. Les pots 5 , ainsi qu'un exemplaire de la jatte 3-1 et du pot 3-8 sont les seules formes associées à ce groupe.

\section{Groupe 4 (GT4)}

Comme pour le groupe précédent, il s'agit d'un groupe individualisé car importé, mais à rapprocher de GT1. Tous les tessons appartiennent à une céramique à parois fines de type « coquille d'œuf ». Les parois sont rarement supérieures à $0,2 \mathrm{~cm}$ d'épaisseur. La pâte est de teinte rose orange, à texture assez fine. Le gobelet 1 est l'unique production présentant cette pâte. Les comparaisons de forme et de pâte indiquent une provenance de la région sarthoise (groupe de Saint-Jean-de-la-Motte) (Husi, 2003, p. 30 ; Guilleux, 1978).

\section{Groupe 5 (GT5)}

21 L'élément caractéristique de ces pâtes est le grésage total des récipients. Selon la teinte des argiles, les grès sont à subdiviser en quatre lots de provenances différentes: Normandie, Beauvaisis et vallée du Rhin.

22 Groupe 5-1: grès de teinte grise, beige ou brun caramel en tranche et gris brun à gris foncé en surface. Les cassures sont nettes, les inclusions ayant fondu dans la masse. Ce groupe renferme l'essentiel des tessons. Importé de la région du Domfrontais (BasseNormandie), il correspond aux formes de pots 1-1 et 1-2 (fig. 9).

Figure 9 : Vue d'un pot à beurre en grès du Domfrontais groupe 5-1. Cliché H. Paitier, Inrap. Figure 9: View of a hammer-headed jug in stoneware of groups 5-1 (from Domfront).

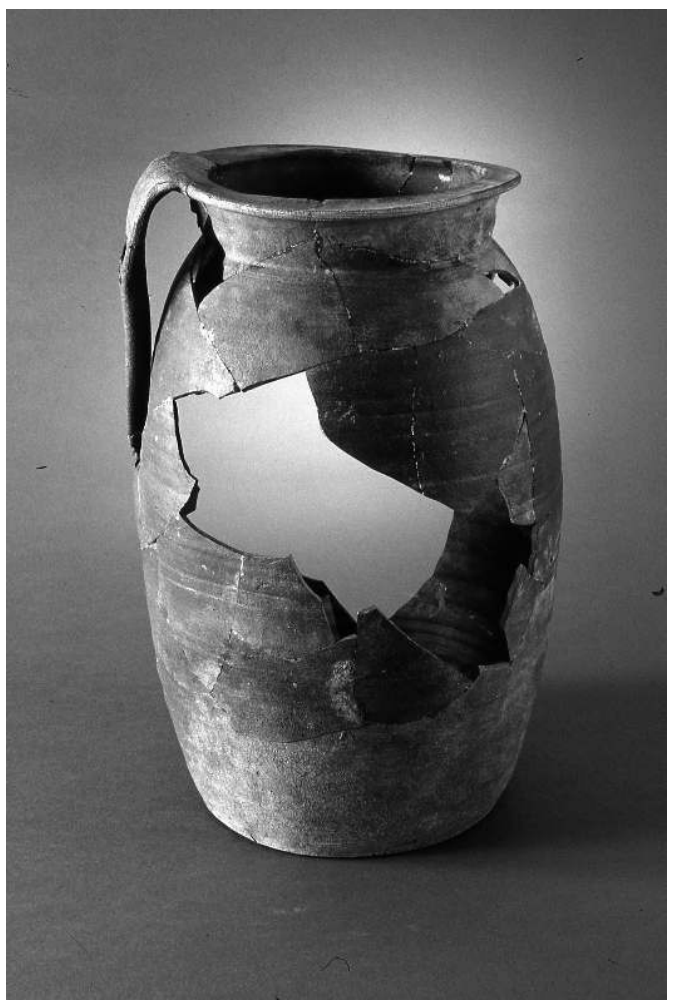


Groupe 5-2: grès de teinte rouge et à inclusions invisibles à l'œil. Les exemplaires moins nombreux de ce groupe appartiennent exclusivement au pot 1 en provenance des ateliers du Bessin-Cotentin.

Groupe 5-3: grès à pâte beige clair et surface beige à brun clair. Les quelques exemplaires recueillis (27 tessons) sont surtout des tessons de coupe 2-2 et de gourde 1-2. Ils sont originaires des ateliers du Beauvaisis.

Groupe 5-4 : grès à pâte claire (gris beige) et à surface grise, flammée. Certaines zones sont recouvertes extérieurement d'une glaçure transparente incolore et brillante. La face externe présente des teintes colorées bleu et violet. Les deux tessons concernés appartiennent à des récipients de type pichet 8, produits dans la vallée du Rhin (ateliers de Cologne, Raeren et Westerwald) (Hurst et al., 1986, p. 177-180).

\section{Groupe 6 (GT6)}

26 Ce lot regroupe les tessons à pâte de teinte rosée et à texture assez fine. Elle est recouverte sur les deux faces d'un émail blanc épais et couvrant avec des éléments de décor colorés (jaune, orange, vert, lie-de-vin et bleu). Cette production est une faïence dont la provenance n'est pas identifiée.

\section{Groupe 7 (GT7)}

27 La pâte de teinte beige possède des inclusions assez abondantes de quartz gris et blanc mais de petite granulométrie. La surface externe conserve les traces d'un engobe assez fin de teinte blanc à beige. Les fragments sont généralement très cuits et sonores. Cette pâte est suffisamment caractéristique pour permettre d'attribuer les tessons de panse à la forme amphore 1. Produites en Espagne, elles servent au commerce de l'huile de Bétique (Hurst et al., 1986, p. 65-67 ; Blieck, 1985, p. 34-37).

\section{Groupe 8 (GT8)}

Ce lot est un sous-groupe de la céramique commune, caractérisé par une pâte claire et une texture grossière riche en grains de quartz blanc et en paillettes de mica. Les tessons sont toujours assez épais ( $1 \mathrm{~cm}$ de moyenne), marqués par des décors à cordons digités. Une partie des récipients est plus ou moins grossièrement modelée. Ce groupe est exclusivement rattaché au vase réserve 1 et à quelques exemplaires uniques (terrine 1). Cette pâte provient peut-être des ateliers de Landéan (35) (Fichet de Clairfontaine, 1996, p. 23 ; Bucur et al., 1984, p. 169-219).

\section{Le catalogue}

La classification s'établit en trois groupes: formes fermées, formes ouvertes et ustensiles. Cette classification portant sur l'ensemble des lots médiévaux et modernes du site, la succession des numéros d'identification peut présenter des manques dans cette étude, correspondant aux formes issues des autres lots non évoqués ici. Chaque type individualisé est alors mentionné par une rubrique «nom» correspondant à la forme générique et par un numéro en chiffres arabes pour le type. Un second numéro lui est adjoint dans le cas des variantes (au besoin, une lettre pour les variations secondaires). Le descriptif indique toujours, dans le même ordre, les aspects marquants 
$\mathrm{du}$ récipient, ses dimensions courantes, les contextes de provenance et le nombre d'exemplaires rencontrés, le groupe technique et l'origine de la production quand elle est connue. Les individus retenus dans la typologie (et l'illustration) correspondent aux récipients les plus complets des formes individualisées, sans inclure les exemplaires uniques et incomplets. Les autres formes fragmentaires sont décrites en référence à des récipients connus dans d'autres contextes régionaux, à l'image ceux du château du Guildo (Beuchet et al., 2004). L'ensemble du mobilier des lots L1 et D2 renferme 46 formes distinctes, réparties en 25 formes fermées, 18 formes ouvertes et 4 ustensiles (fig. 10, 12, 13, 15, 17 et 18).

\section{Les récipients fermés}

Cette rubrique regroupe les récipients de formes variées qui ont pour constante une ouverture proche du diamètre maximum de la panse (fig. 10).

Figure 10 : Typologie des formes rencontrées. Vases fermés. Infographie : F. Labaune-Jean, Inrap. Figure 10: Typology of the met forms. Closed vases.

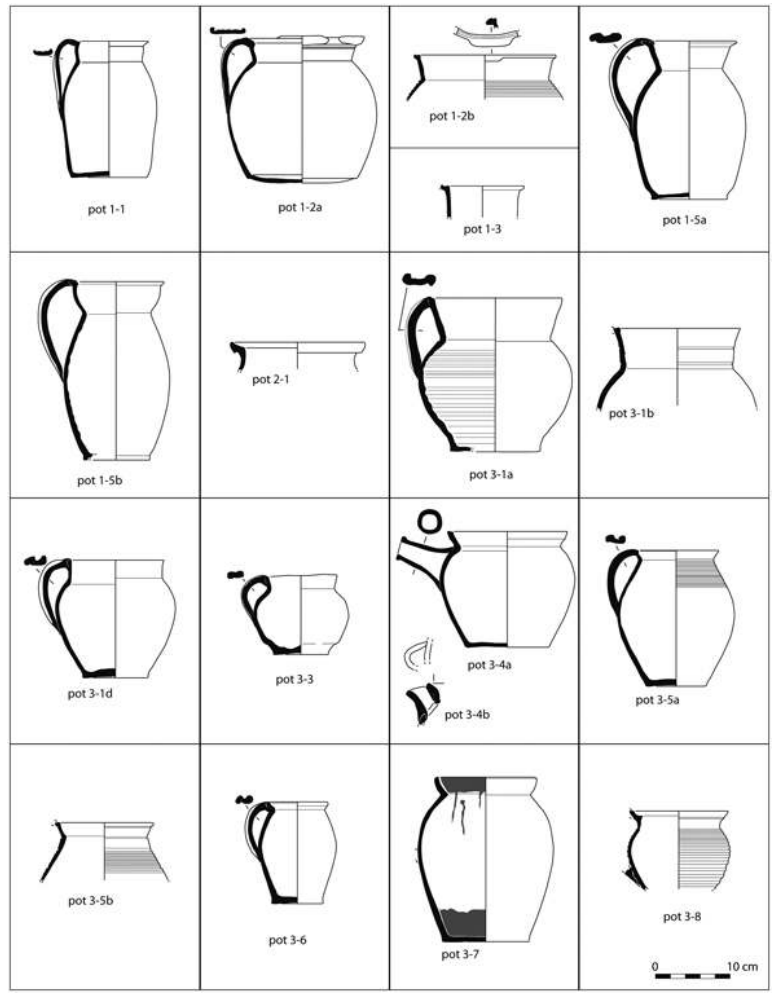

Pot 1

Récipient de forme élancée à col vertical court et lèvre en méplat horizontal. Réalisé en pâte du groupe GT5-1 et 2 et GT1, il comprend cinq variantes.

Pot 1-1: rebord en méplat horizontal, concave avec bourrelet rentrant vers l'intérieur. Le col est court, droit ou légèrement évasé. La jonction col-panse est souvent soulignée d'un bourrelet. La panse présente un profil élancé, ovoïde dans la moitié supérieure et cylindrique dans la partie basse. La base se délimite par un fond plat assez épais. Une anse large à face externe striée verticalement est appliquée sur la lèvre et le milieu de 
la panse. Diamètre de 12 à $18 \mathrm{~cm}$. Hauteur de 16 à $33 \mathrm{~cm}$. 56 exemplaires. GT5. Contextes : L1 et D2.

Pot 1-2 : rebord en méplat horizontal ou oblique et col court vertical. La différence tient à la présence de 1 à 3 tenons horizontaux, appliqués sur le rebord externe de la lèvre. La panse ovoïde est de hauteur moyenne (environ $20 \mathrm{~cm}$ ). Il faut signaler une anse verticale très large, striée longitudinalement, dont les rebords sont repliés sur le dessus. Elle se fixe sur le haut de la lèvre et le milieu de la panse. Le récipient repose sur un fond assez épais, légèrement convexe. La variante $1-2 b$ possède des tenons moins développés et un décor de cannelures sur l'épaule (2 exemplaires. Contextes : F1 et D2). Diamètre de 15 à $19 \mathrm{~cm}$. Hauteur complète conservée: $23 \mathrm{~cm} .17$ exemplaires. GT5. Contexte : D2.

Pot 1-3 : rebord en bourrelet court à méplat supérieur concave. L'embouchure est de plus petit diamètre que celle des précédents (9 et $11 \mathrm{~cm}$ ). Une attache d'anse figure sur le rebord de la lèvre. GT5. 1 exemplaire. Contexte : L1.

Pot 1-5: panse assez haute, rétrécie par un col évasé (1-5a) ou courbe (1-5b), qui se termine par une lèvre en méplat mouluré. La préhension se fait grâce à une anse plate à sillon. La base possède un petit bourrelet externe et un fond légèrement rentrant. Diamètre de 12 à $15 \mathrm{~cm}$. Hauteur : $21 \mathrm{~cm} .135$ exemplaires. GT1. Contextes : L1 et D2.

Pot 2

Pot 2-1 à embouchure constituée d'une lèvre éversée en bandeau, à pan interne concave et col court. Il est généralement doté d'une panse ovoïde à surface externe cannelée, reposant sur un fond plat ou lenticulaire. Ce récipient correspond aux oules de type $1 \mathrm{a}$ et $1 \mathrm{~b}$ produites dans l'atelier de Fontenay à Chartres-de-Bretagne aux XII ${ }^{\mathrm{e}}$ et $\mathrm{XIII}^{\mathrm{e}}$ siècles. Il est juste évoqué ici, car en présence résiduelle parmi le mobilier issu des latrines L1 (2 exemplaires. Contexte : L1).

Pot 3

Réalisé presque exclusivement en pâte GT1, ce pot, également appelé coquemar, est caractérisé par une lèvre placée dans le prolongement d'un col vertical ou légèrement éversé et une panse globulaire ou ovoïde reposant sur un fond plat. La préhension se fait au moyen d'une anse plate prenant appui sur la lèvre et l'épaule ou par un manche creux fixé sur l'épaulement. Les teintes vont du brun clair au gris foncé. Les variantes reposent sur le profil de la panse et l'aspect de la lèvre (fig. 11). 
Figure 11 : Vue des récipients intacts, complets et des vases déformés. Cliché H. Paitier, Inrap. Figure 11: View of the intact, complete bowls and the deformed vases.

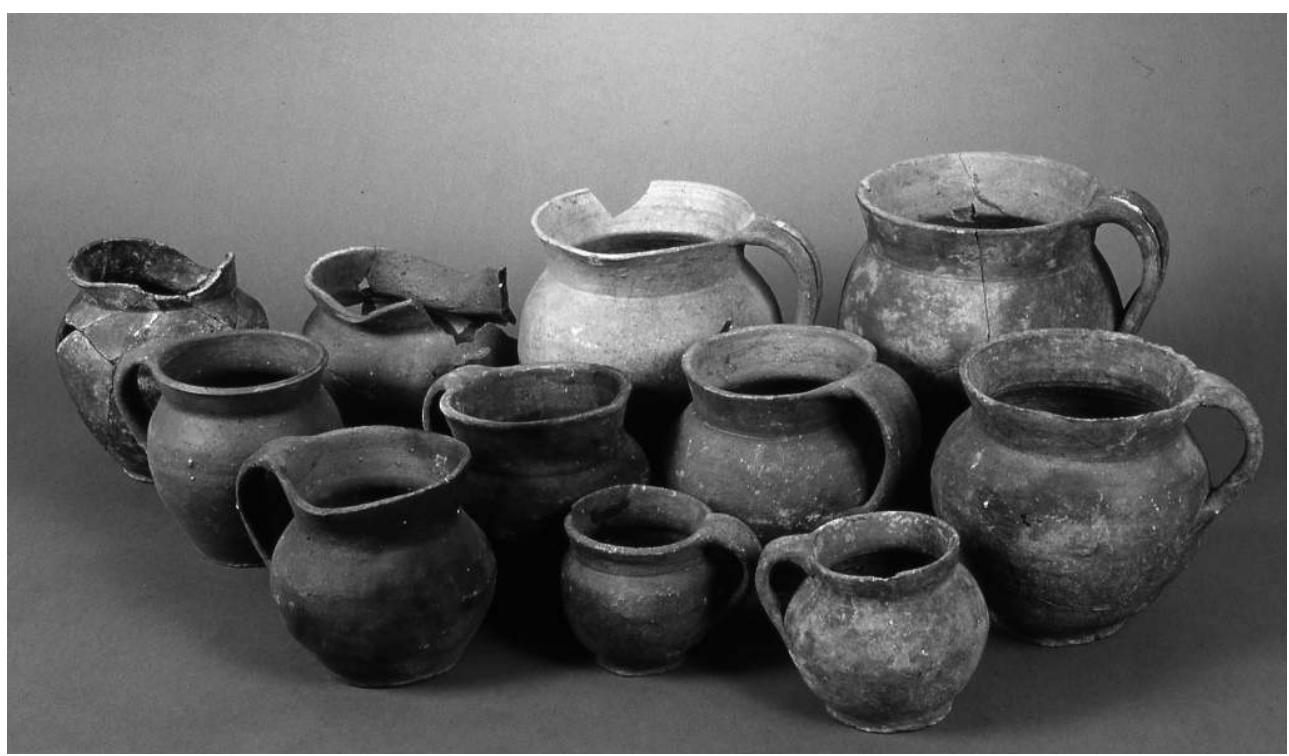

Pot 3-1: le col forme un bandeau assez haut, légèrement évasé, terminé par une lèvre à pan oblique ou arrondi. Diamètre et hauteur de 10 à $24 \mathrm{~cm}$. GT1. Contextes : L1 et D2. La variante 3-1b présente des cannelures sur le col (5 exemplaires en L1) et le type 3-1c est doté d'une lèvre courte en bourrelet angulaire (65 exemplaires en L1 et D2). La variante 3-1d se compose d'une panse toujours ovoïde avec un col moyen et vertical terminé par une lèvre confondue dont l'extrémité est dotée d'une fine cannelure. Diamètres de 8 à $23 \mathrm{~cm}$ et hauteur de 15 à $23 \mathrm{~cm} .6321$ exemplaires. Contexte : D2.

Pot 3-3 : ce pot est souvent de petite taille, à panse globulaire et lèvre courte arrondie. Diamètres de 6 à $14 \mathrm{~cm}$. Hauteur de 8 à $15 \mathrm{~cm} .6$ exemplaires. GT1. Contextes : L1 et D2.

Pot 3-4: ce lot regroupe les fragments dotés d'un manche creux à la place d'une anse. Diamètre de 14 à $19 \mathrm{~cm}$. Hauteur 14/15 cm. 2 exemplaires. GT1. Contextes : L1 et D2. La variante $3-4 \mathrm{~b}$ renferme un seul exemplaire à bec verseur ponté (contexte L1).

Pot 3-5: il se distingue par une lèvre confondue avec un col court éversé et une panse globulaire dont le tiers supérieur peut être décoré. Il est doté d'une panse ovoïde, d'un fond plat et d'une anse plate à sillon. GT1. (Le comptage précis entre les deux types n'a pu être réalisé dans le cadre de la post-fouille. Au total, 1122 exemplaires sont donc à répartir entre les types pot 3-5 et pot 3-6). Le type 3-5a présente un décor systématique à base des fines stries horizontales disposées sur le tiers supérieur de la panse (Contextes: L1 et D2). La forme 3-5b est pourvue d'un léger bourrelet jouxté d'une cannelure au niveau de la lèvre. Diamètre de 10 à $16 \mathrm{~cm}$ (Contextes : L1).

Pot 3-6 : le récipient correspond à une version du type 3-5 au profil plus élancé et sans décor strié. Diamètre de 7 à $14 \mathrm{~cm}$. Hauteur de 13 à $26 \mathrm{~cm}$. À part quelques grands modules, la plupart de ces récipients est de petite taille. GT1. Contextes : L1 et D2.

Pot 3-7 : ce vase de taille moyenne est toujours glaçuré sur le fond interne et la lèvre. Cette dernière, confondue avec le col, est haute et à pan interne concave. Diamètre de 13 à $15 \mathrm{~cm}$. Hauteur conservée : $22 \mathrm{~cm}$. 10 exemplaires. GT2-1. Contextes : L1 et D2.

Pot 3-8: de petite taille, ce pot possède une lèvre courte, éversée à extrémité plate ou arrondie avec un pan interne concave formant une gouttière prononcée. Le col est absent. La panse ovoïde ou globulaire est ornée de stries marquées sur la face externe. 
Une anse de section quadrangulaire vient se fixer à la jonction lèvre-panse et dans la partie basse de cette dernière. La caractéristique principale repose sur la pâte riche en feldspath, de teinte orange à gris bleuté. Cette production d'aspect comparable à celle de la céramique rose bleue de Laval (GT3) ne figure cependant pas parmi les formes recensées de ces ateliers. 2 exemplaires. Contexte : D2.

Pot 3-9: dérivé des coquemars, ce récipient comprend une panse globulaire à fond plat, terminée par une lèvre éversée à méplat interne oblique et face externe dotée d'un double bourrelet séparé par une cannelure. Des traces de glaçure subsistent sur le rebord interne (groupe 2). Une anse plate à sillon vient se fixer sur la lèvre qu'elle englobe et sur la moitié inférieure de la panse. Diamètre de 13 à $16 \mathrm{~cm}$. Hauteur de 13 à $17 \mathrm{~cm} .1$ exemplaire. GT2-1. Contexte : D2 (fig. 12).

Figure 12 : Typologie des formes rencontrées. Vases fermés (suite). Infographie : F. Labaune-Jean, Inrap.

Figure 12: Typology of the met forms. Closed vase.

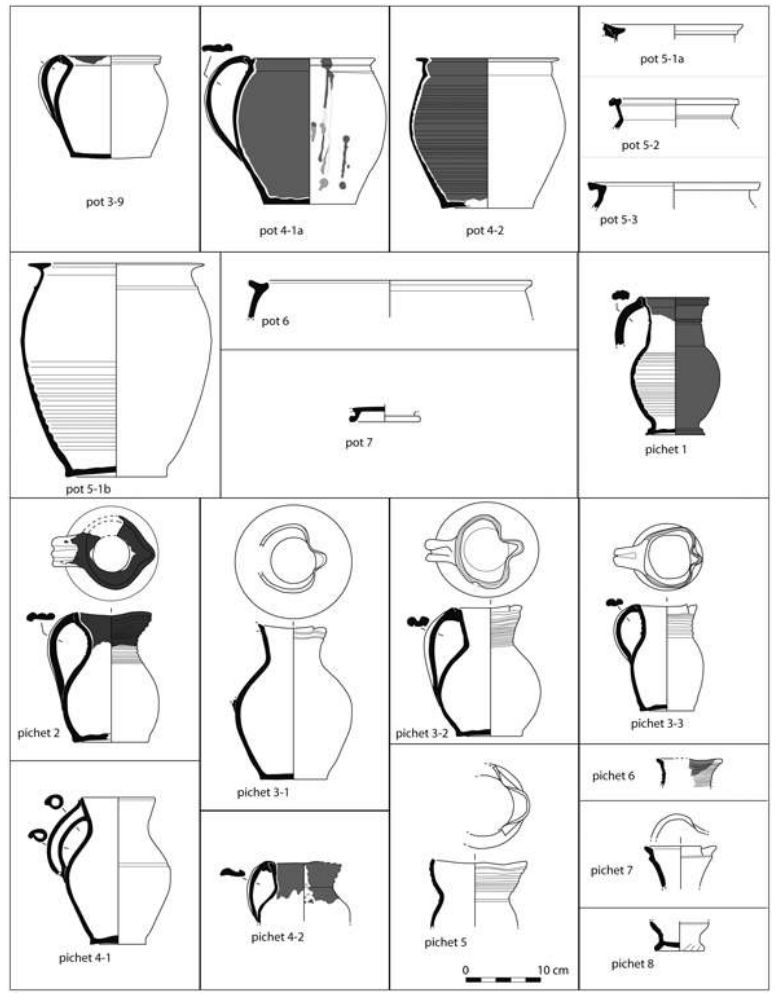

Pot 4

Cette production (type 4-1) montre une lèvre courte en collerette horizontale, associée à un col court et une panse ovoïde reposant sur un fond plat. Une large anse plate permet la préhension. La surface interne est glaçurée sur toute la hauteur du récipient (GT2-1). Dans les contextes plus récents (xvII e siècle), il pourra être pourvu de trois pieds fixés sous le fond. Diamètre : 17,5 cm. Hauteur : 19,5 cm. 7 exemplaires. Contexte : D2. La variante 4-2 est distinguée par les parois très fines des récipients, la forme restant similaire à la précédente. Le récipient est réalisé uniquement dans une pâte de teinte très claire, presque blanche (GT2-3). Une glaçure homogène et fine de teinte 
verte recouvre entièrement l'intérieur. Diamètre de 12 à $19 \mathrm{~cm} .23$ exemplaires. Contexte : D2.

Pot 5 annulaire, sa panse globulaire et sa lèvre en collerette arrondie, jouxtée d'une anse en boucle. Les fragments sont tous du GT7 et correspondent à des pots de chambre en faïence blanche. Ils sont signalés ici mais constituent un élément (2 tessons) correspondant à une intrusion résiduelle des couches supérieures au dépotoir D2.

\section{Pichet 1}

50 Ce pichet (variante 1-1) comprend une ouverture assez étroite définie par une lèvre en bandeau mouluré, placée dans le prolongement d'un col cylindrique assez haut. La panse ovoïde repose sur une base rétrécie et un fond évasé légèrement rentrant. La surface externe et le haut de la lèvre interne sont pourvus d'une glaçure couvrante. Une anse quadrangulaire moulurée permet la préhension. 5 exemplaires. GT2-4. Contextes : L1 et D2.

\section{Pichet 2}

51 Les exemplaires complets de type 2-1 montrent un profil à panse globulaire surbaissée, reposant sur un fond plat. Le col, rétréci et concave, est orné de fines stries horizontales avant de s'évaser pour constituer la lèvre verticale courte à moulure externe. La glaçure verte est apposée sur le rebord interne et externe. Un aménagement forme le bec verseur en opposition avec l'anse plate à double sillon. 18 exemplaires. GT2-1. Contexte : D2. 


\section{Pichet 3}

Réalisé en céramique commune sans revêtement, ce type se compose d'une panse ovoïde ou globulaire, à fond plat. Le col cylindrique, légèrement évasé, est prolongé par une petite lèvre effilée et moulurée. Un pincement permet d'aménager le bec verseur. Une anse se fixe sur la lèvre et au milieu de la panse. Les variantes se basent sur la présence de stries sous la lèvre (3-1) ou de cannelures sur toute la hauteur du col (3-2). 602 exemplaires. GT1. Contexte : D2.

\section{Pichet 4}

Ce type se différencie par une lèvre à extrémité arrondie placée dans l'axe du col évasé. Le bord ne présente aucun bec verseur. Il est doté d'une anse creuse. Les récipients retrouvés possèdent une pâte du groupe GT1. 3 exemplaires. Contextes : L1 et D2. La variante 4-2 est pourvue d'un bec verseur et de glaçure. 1 exemplaire. GT2-1. Contexte : D2.

\section{Pichet 5}

54 Ces pichets (variante 5-1) possèdent un large col convexe, strié, terminé par une lèvre arrondie dotée d'un large bec verseur. 9 exemplaires. GT1. Contexte : L1.

\section{Pichet 6}

Seule la lèvre permet de faire la distinction du pichet 6-1 avec le pichet 3-2. Elle se compose d'un petit méplat court et oblique. 1 exemplaire. GT2-1. Contexte : L1.

\section{Pichet 7-1}

Le profil de ce pichet montre une lèvre en méplat oblique et un bec pincé. La jonction avec le col est soulignée par une carène prononcée. Le haut du col est oblique et assez étroit. 22 exemplaires. GT1. Contexte : L1.

\section{Pichet 8-1}

Grâce à la pâte grésée de teinte claire, un fond et un fragment de panse ont pu être identifiés comme des productions des ateliers de la vallée du Rhin. Ce pichet décoré ou bellarmine fait partie du groupe GT5-4. 1 exemplaire. Contexte : D2.

\section{Cruche 1}

La partie haute du vase 1-1 est délimitée par un col cylindrique et une lèvre éversée en bourrelet de section quadrangulaire mouluré. Le départ de panse indique une panse de grande contenance. 6 exemplaires. GT1. Contextes : L1 et D2 (fig. 13). 
Figure 13 : Typologie des formes rencontrées. Vases fermés (suite et fin). Infographie : F. LabauneJean, Inrap.

Figure 13: Typology of the met forms. Closed vases(end).

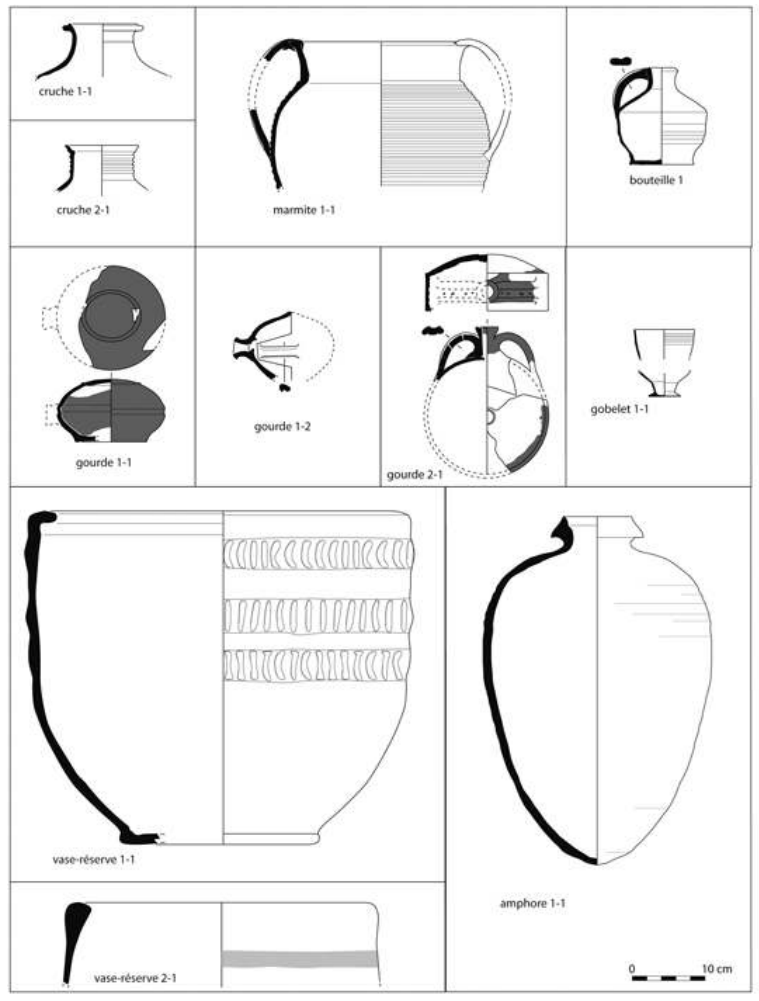

Cruche 2

Le profil 2-1 est similaire au type cruche 1-1, avec un col cylindrique entièrement cannelé et une petite lèvre effilée à gouttière interne. La pâte présente de grandes similitudes avec le groupe GT3. 2 exemplaires. Contextes : L1 et D2.

\section{Marmite 1}

De grande contenance, ces récipients type marmite 1-1) se caractérisent par une large ouverture à lèvre en méplat horizontal, un col court et droit ainsi qu'une panse globulaire entièrement cannelée sur la face externe reposant sur un fond plat. Deux anses larges sont placées en vis-à-vis sur le bord et le milieu de la panse. Ils sont tous en pâte du groupe GT1. Diamètre de 18 à $26 \mathrm{~cm} .20$ exemplaires. GT1. Contextes : L1 et D2.

\section{Bouteille 1}

61 Cet unicum (bouteille 1-1) est réalisé dans une pâte commune particulière (riche en inclusions de feldspath) sans provenance établie. La base large et plate se poursuit par une panse cylindrique marquée par une carène au niveau de l'épaulement et de la transition avec le goulot étroit. La lèvre est légèrement épaissie, à pan externe biseauté. 1 exemplaire. GT1-2. Contexte : D2. (fig. 14) 
Figure 14 : Vue d'une bouteille intacte à pâte granuleuse. Cliché H. Paitier, Inrap. Figure 14: View of an intact bottle with granular fabric.

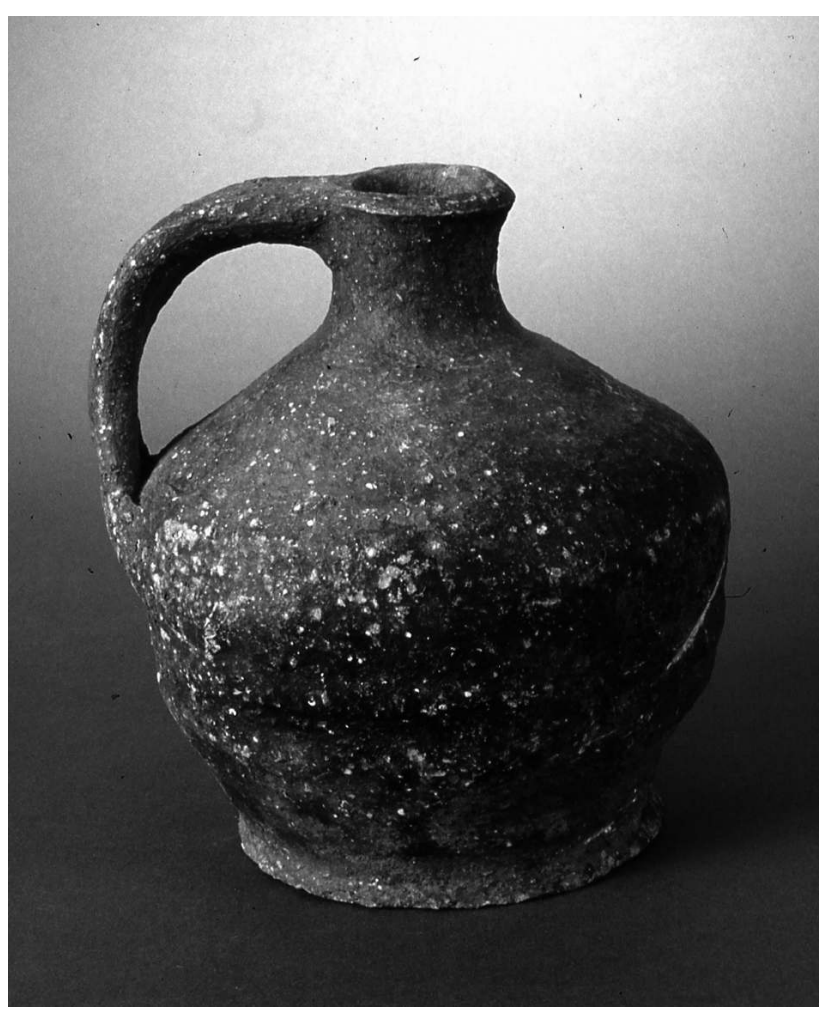

\section{Gourde 1}

Destinée au transport des liquides, elle se compose d'une panse circulaire fermée avec une face bombée et une autre plate sur laquelle on peut poser le récipient. Un goulot jouxté de deux petites anses venait se placer sur la tranche. La variante 1-1 présente une pâte du groupe GT2-4 (1 exemplaire. Contexte: D2), alors que le type 1-2, pourvu d'anses plus grandes, est en pâte du groupe GT5-3 (Beauvaisis). 3 exemplaires. Contexte : D2.

\section{Gourde 2}

Directement copiée des modèles en cuir, cette gourde 2-1 possède une forme de tambourin à face bombée. Le goulot court, à embouchure évasée en trompette, est jouxté de deux longues anses plates. Ils sont placés sur la partie en anneau plat du récipient. Tous les fragments rencontrés sont dotés d'une glaçure verte (GT2-1). 3 exemplaires. Contexte : D2.

\section{Gobelet 1}

Réalisé en pâte du groupe 4 , ce petit gobelet 1-1 possède une vasque tronconique terminée par une lèvre arrondie placée dans le prolongement de la paroi. Cette dernière n'excède jamais $2 \mathrm{~mm}$ d'épaisseur. La base est généralement étranglée à fond plat évasé et découpée à la ficelle. 15 exemplaires. GT4. Contexte : L1 et D2. 


\section{Vase réserve 1 et 2} épaisses, de cordons décorés d'impressions digitées ou d'incisions obliques. Les lèvres parfois simplement arrondies dans le prolongement du corps (variante 2-1, 5 exemplaires. GT8. Contexte: L1), sont généralement repliées en méplat horizontal interne (variante 1-1, 11 exemplaires. GT8. Contexte : L1, D2). Les exemplaires voient leur forme complétée par une panse cylindrique de fort diamètre et un fond plat formant un petit bourrelet externe.

\section{Amphore 1}

Bien qu'anecdotique, ce récipient (amphore 1-1) est à signaler dans le dépotoir D2, sous forme de 13 fragments de panse. Réalisé dans une panse de groupe GT7, il présente généralement une panse allongée et cannelée, dotée d'un fond en pointe et d'un goulot étranglé terminé par une lèvre de section triangulaire. 1 exemplaire. GT7. Contexte : D2.

\section{Les récipients ouverts}

Cet ensemble regroupe les récipients dont l'ouverture est plus large que le reste du corps. La plupart de ces types sert au service des aliments. (fig. 15 et 17)

Figure 15 : Typologie des formes ouvertes rencontrées. Infographie : F. Labaune-Jean, Inrap. Figure 15: Typology of open forms.

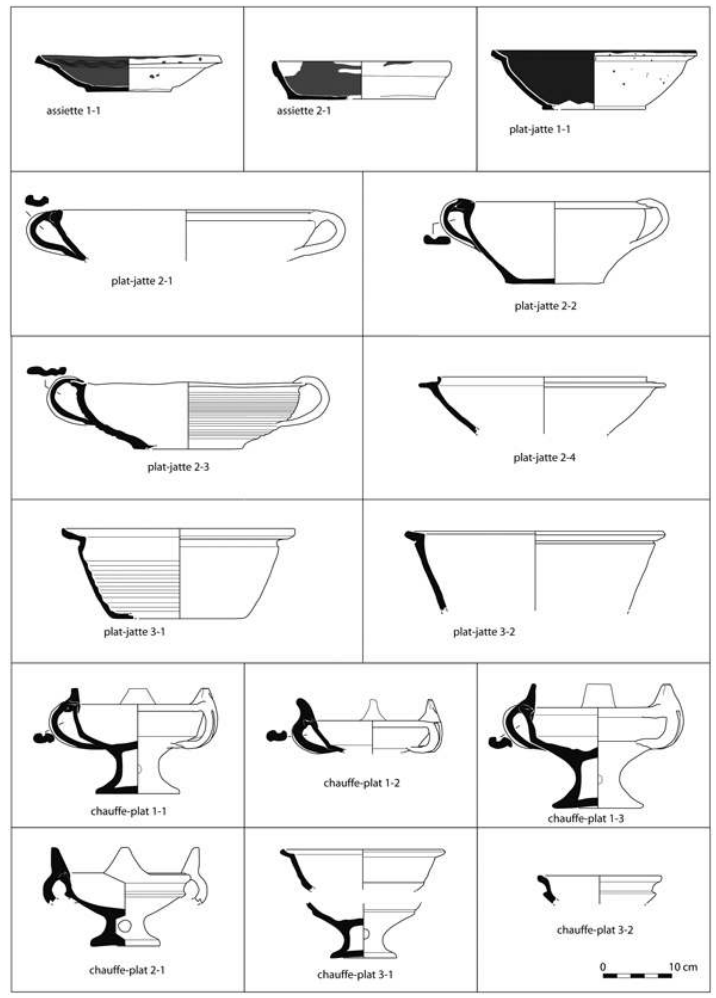




\section{Assiette 1}

Constituée d'une panse évasée et d'un fond large et plat, cette assiette 1-1 se définit par sa lèvre en large méplat éversé, terminé par un bourrelet rond ou triangulaire. La surface interne du récipient est toujours pourvue d'une glaçure couvrante de teinte jaune à vert uniforme ou marbrée (groupe GT2-1). Un décor incisé (souvent ondé) peut être effectué avant glaçure sur le méplat interne. 20 exemplaires. Contextes : L1, D2.

\section{Assiette 2}

Cette assiette ou écuelle 2-1 possède un large fond plat, une panse oblique courte et une lèvre en bourrelet externe étiré. Les deux exemplaires mis au jour correspondent au sous-groupe GT2-3: pâte claire, à surface granuleuse et glaçure vert moyen. 2 exemplaires. GT2-3. Contexte : D2.

\section{Plat - jatte 1}

Ce plat 1-1 est le pendant de l'assiette 1-1 mais avec une vasque plus profonde et une lèvre à méplat souvent moins importante. 3 exemplaires. GT2. Contextes : L1, D2.

\section{Plat - jatte 2}

71 Cette forme est dotée d'une large vasque évasée, terminée par une lèvre en bourrelet de section triangulaire. La préhension se fait au moyen de deux anses plates assez courtes placées en vis-à-vis sur la lèvre et le haut de la panse. Une version sans anse existe. La surface externe peut être lisse ou cannelée horizontalement sur toute la hauteur.

Plat-jatte 2-1: se compose d'une panse large, évasée à l'oblique et peu profonde. La lèvre est en bourrelet de section triangulaire. 2 exemplaires. GT1. Contextes: L1. Proche de la forme précédente, les types 2-2 à 2-4 se distingue par une lèvre éversée en collerette. Trois variantes existent suivant la lèvre. Diamètre de 30 à $34 \mathrm{~cm}$. 65 exemplaires. GT1. Contexte : D2.

Plat-jatte 2-2 : lèvre en crochet épais souligné d'une cannelure vers l'intérieur.

Plat-jatte 2-3 : lèvre éversée en méplat à pan concave. Panse cannelée

Plat-jatte 2-4: lèvre éversée en collerette horizontale, avec une moulure interne très débordante. Vasque conique.

\section{Plat-jatte 3}

76 Ce vase possède une large panse de profil tronconique, reposant sur un fond plat et terminé par une lèvre en large méplat éversé à l'oblique (type 3-1). Les stries de tournage sont très visibles sur l'intérieur. Une cannelure marquée marque la transition avec la vasque conique profonde. 2 exemplaires. GT1. Contexte : L1 et D2.

77 La version type 3-2 montre une lèvre plus courte et placée à l'horizontale, dans le prolongement d'une vasque tronconique lisse. GT1. Contexte : D2. 


\section{Chauffe-plat 1} présente un fond évasé et plat, prolongé par un corps creux et cylindrique, pourvu d'une perforation. La vasque de profil caréné, assez peu profonde, est terminée par une lèvre éversée en méplat court. Trois anses plates à sillon sont réparties sur le pourtour du récipient. Trois tenons de profil pyramidal sont placés au-dessus de ces anses. La variante 1-2 possède une lèvre plus développée et des tenons triangulaires très effilés. 21 exemplaires. GT1. Contextes : L1 et D2. Ce type 1-3 se caractérise surtout par sa lèvre de section triangulaire à pan supérieur concave. 29 exemplaires. GT1. Contexte : D2.

Figure 16: Vue d'un chauffe-plat en céramique du groupe technique 1. Cliché H. Paitier, Inrap. Figure 16: View of a chafing dish (technical group 1).

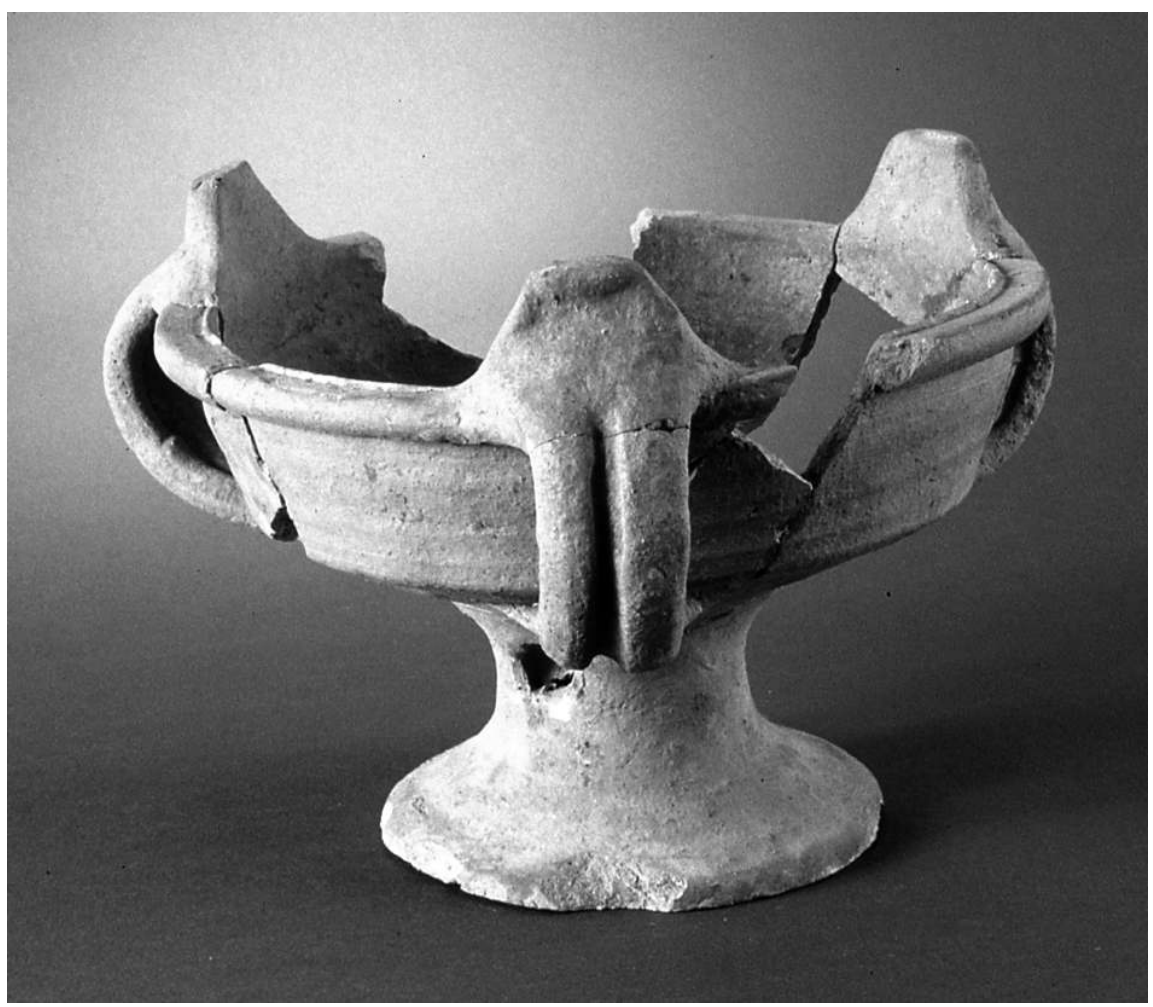

\section{Chauffe-plat 3}

Ce type présente le même profil que les précédents avec une lèvre en méplat concave, simplement éversé vers l'extérieur. 3 exemplaires. GT1. Contexte : L1. La variante 3-2 possède une carène très marquée. GT1. 1 exemplaire. Contexte : L1.

\section{Poêle 2-1}

Ce récipient est caractérisé par une vasque carénée, une lèvre éversée en méplat horizontal et un manche cylindrique creux pour la préhension. 2 exemplaires. GT2-1. Contexte : D2. (fig. 17) 
Figure 17 : Typologie des formes ouvertes rencontrées (suite). Infographie : F. Labaune-Jean, Inrap. Figure 17: Typology of open forms (next).

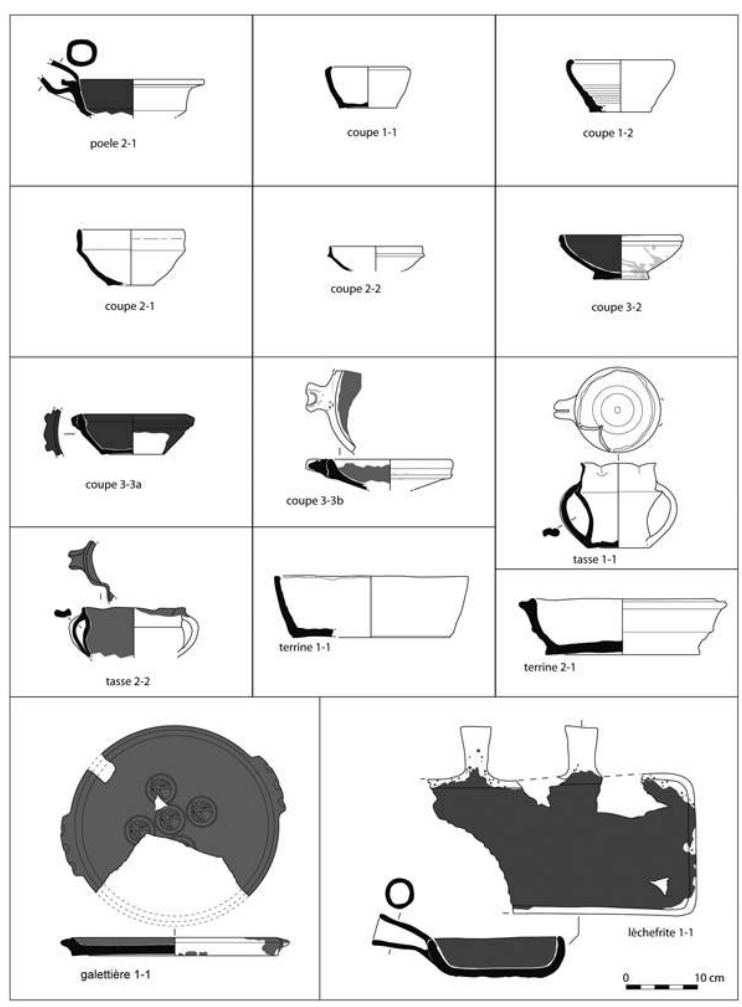

Coupe 1

81 Cette petite forme se compose d'une vasque tronconique, reposant sur un fond plat et prolongé par une lèvre biseautée rentrante. 1 exemplaire. GT1. Contexte: D2. Plus évasée que la précédente, la vasque de la variante 1-2 se termine par une courbe et une lèvre ronde rentrante. Les exemplaires sont tous en pâte du groupe GT1. 3 exemplaires. Contexte : L1.

\section{Coupe 2}

82 Cette coupe possède une vasque conique à fond plat. Après une carène angulaire, la paroi devient droite et se termine par une lèvre arrondie. La variante 2-2 à lèvre rentrante correspond aux productions en grès du Beauvaisis. 2 exemplaires. GT5-3. Contexte : D2.

\section{Coupe 3}

Cette forme présente une vasque courbe reposant sur un pied court mouluré à fond plat.

Coupe 3-2. La forme se distingue par une cannelure assez prononcée, placée sous l'arrondi de la lèvre. Elle ne semble pas être pourvue d'anse. 5 exemplaires. GT2-1. Contextes : L1 et D2.

Coupe 3-3a: la vasque oblique n'est pas très haute. La lèvre triangulaire à pan interne concave est soulignée par une cannelure externe. Deux tenons digités, peu développés, 
sont collés près de la lèvre pour symboliser les anses. 1 exemplaire. GT2-1. Contexte : D2.

Coupe 3-3b : la vasque très oblique et peu profonde se prolonge par une lèvre épaisse verticale de section triangulaire. La face externe est ornée d'une large cannelure, au dessus de la carène de transition. Une petite poignée pincée de forme rectangulaire est placée contre la lèvre. 1 exemplaire. GT2-1. Contexte : D2.

Tasse 1

Réalisée en pâte très cuite (GT1), cette tasse 1-1 se compose d'une panse cylindrique, s'évasant légèrement jusqu'à une carène angulaire. Verticale ensuite, elle est prolongée par une extrémité arrondie constituant la lèvre. Elle est pincée régulièrement vers l'intérieur de manière à former une ouverture à 4 lobes. La base plate est délimitée par un fond peu rentrant. Deux anses plates à sillon se fixent sur la carène et à la base du vase. 14 exemplaires. Contexte : L1.

\section{Tasse 2}

Très proche du type 1 , elle s'en distingue par une panse globulaire dans la partie basse ainsi que par des anses fixées sur la lèvre et le milieu de la panse. Seule la variante 2-2 est représentée dans ces deux contextes. Le vase est formé d'une panse courbe, à profil en $\mathrm{S}$ étiré. La lèvre à extrémité arrondie ou plate est régulièrement pincée vers l'intérieur de manière à former une ouverture polylobée. Deux petites anses sont appliquées sous la lèvre et sur la panse. La surface interne est entièrement recouverte d'une glaçure verte. 3 exemplaires. GT2-1. Contexte : L1 et D2.

\section{Terrine 1}

Les parois verticales du type 1-1, légèrement courbes de ce récipient délimitent une vasque largement ouverte et peu profonde, reposant sur un fond plat. La lèvre placée dans le prolongement est légèrement arrondie et forme un petit bourrelet interne irrégulier. 1 exemplaire. GT8. Contextes : L1.

\section{Terrine 2}

Ce type 2-1 se compose d'une panse courbe carénée, d'un fond épais légèrement débordant et d'une lèvre de section triangulaire à pan supérieur concave. Elle a aussi pu servir de couvercle. 1 exemplaire. GT1. Contexte : C2.

\section{Galettière 1}

91 Cette forme particulière (type 1-1) présente un disque de grand diamètre, assez épais, recourbé sur une faible hauteur à sa périphérie pour former un rebord vertical à lèvre biseautée. Deux tenons larges mais peu développés sont disposés en vis-à-vis sur le bord. Un décor centré à base de sceaux est imprimé à l'intérieur du récipient avant cuisson et application de la glaçure homogène couvrant toute la surface interne. 2 exemplaires. GT2-1. Contexte : D2. 


\section{Lèchefrite 1} spécifiques. (fig. 18).

Figure 18 : Typologie des ustensiles. Infographie : F. Labaune-Jean, Inrap. Figure 18: Typology of the accessories.

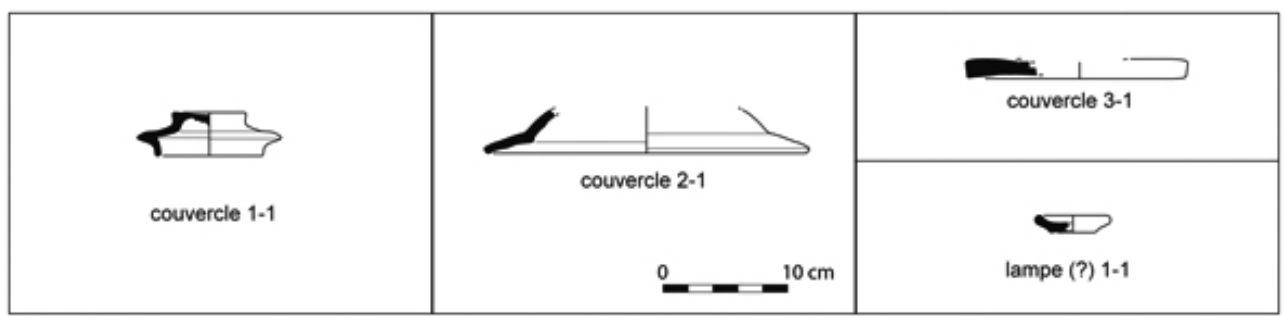

\section{Couvercle 1}

Ce premier type 1-1 présente un système de double lèvre destiné à l'encastrement dans l'ouverture du récipient à clore. La lèvre verticale à extrémité arrondie forme un repli important constituant une collerette externe. Le reste du corps est très oblique, puis se rétrécit sur le fond plat servant à la préhension. Diamètre de 8,5 à $20 \mathrm{~cm} .6$ exemplaires. GT1. Contexte : L1 et D2.

\section{Couvercle 2}

95 Ce second type 2-1 possède un profil plus creux, terminé par une lèvre à méplat éversé en collerette large et pan interne concave. 1 exemplaire. GT1. Contexte : D2.

\section{Couvercle 3}

Ce type 3-1 correspond à la version la plus simple directement copiée des formes en bois. Il se compose d'un disque de terre cuite à tranche droite. 1 exemplaire. GT1. Contexte : D2.

\section{Lampe 1 (?)}

Une petite coupelle (type 1-1) à vasque très évasée terminée par une lèvre arrondie représente un exemplaire unique de petit récipient correspondant peut-être à une lampe (Lefevre, Meyer, 1988). GT1. Contexte : D2. 


\section{L'exploitation des données}

\section{Les provenances}

La fouille de ce site a livré des structures identifiables aussi bien sur le terrain que dans les archives, et des quantités importantes de mobilier riche, varié et homogène. Ces facteurs se conjuguent pour que les lots de mobilier du site de la place Sainte-Anne servent de référence pour l'établissement de cette première typologie des récipients en céramique en usage sur Rennes à l'époque moderne. Le nombre de récipients est suffisamment important dans chaque ensemble pour permettre de dégager certaines associations de formes.

Dans un premier temps, la répartition des tessons (tab.1) montre des représentations très variables selon les groupes techniques. Le tableau présente les comptages par ensemble et par groupe en signalant, à chaque fois, le nombre de tessons avant recollage, sa traduction en pourcentage et le nombre de vases estimés (NMI). Pour les deux lots, le groupe GT1 est prédominant avec près de 87 à $94 \%$ du total des tessons. Tous les vases sont très cuits, voire surcuits avec de nombreux individus à la limite du grésage. Ces productions, malgré l'absence d'analyses, semblent attribuables aux ateliers de Chartres-de-Bretagne. Les mêmes remarques sont valables pour le groupe GT2-1, correspondant aux mêmes pâtes mais avec de la glaçure. L'approvisionnement prioritaire est donc donné aux productions des ateliers proches. Les autres pâtes sont relativement peu représentées, avec un pourcentage inférieur à $2 \%$. (Pour les latrines L1, les grès atteignent $8,4 \%$ sur le nombre total de tessons, mais ce chiffre est à moduler car le mobilier céramique est ici très fragmenté). Cette faible proportion est tout fait justifiée par les formes appartenant surtout à de la vaisselle culinaire. Pour un usage courant, il est normal que les céramiques utilisées soient de productions locales. De plus, il convient de rappeler le contexte hospitalier du secteur, où une vaisselle tout au moins plus coûteuse à l'achat n'est pas de mise.

Tableau 1 : Répartition des tessons en nombre de fragments, en nombre d'individus estimés et en pourcentage par structure de découverte et par groupe technique. F. Labaune-Jean, Inrap. Table 1: Distribution of shards in number of fragments, in number of estimated individuals and in percentage by structure of discovery and by technical group.

\begin{tabular}{|l|l|l|l|l|l|l|l|l|l|l|}
\hline Contexte & & GT1 & GT2 & GT3 & GT4 & GT5 & GT6 & GT7 & GT8 & Total \\
\hline 2512-2540-2702 (L1) & De tessons & 12367 & 220 & 146 & 39 & 1041 & 0 & 0 & 201 & 14014 \\
& (NR) & & & & & & & & & \\
& $\%$ NR & 88,2 & 1,6 & 1,1 & 0,3 & 7,4 & 0 & 0 & 1,4 & 100 \\
\hline & NMI & 580 & 43 & 7 & 10 & 56 & 0 & 0 & 18 & 714 \\
\hline & $\%$ NMI & 81 & 6 & 1 & 1,5 & 7,9 & 0 & 0 & 2,6 & 100 \\
\hline
\end{tabular}




\begin{tabular}{|l|l|l|l|l|l|l|l|l|l|l|}
\hline 2509-2510-2700-2701 (D2) & De tessons & 67625 & 1806 & 1527 & 13 & 872 & 2 & 24 & 88 & 71964 \\
& (NR) & & & & & & & & & \\
\hline & $\%$ NR & 94 & 2,5 & 2,1 & 0,02 & 1,2 & 0,002 & 0,03 & 0,1 & 100 \\
\hline & NMI & 8052 & 124 & 61 & 5 & 47 & 2 & 3 & 21 & 8315 \\
\hline & $\%$ NMI & 96,8 & 1,5 & 0,7 & 0,1 & 0,6 & 0,02 & 0,04 & 0,3 & 100 \\
\hline
\end{tabular}

D'ores et déjà, on note plusieurs zones d'approvisionnement (fig. 19) avec :

- - des céramiques importées au niveau régional proche (dans un rayon de moins de cent de kilomètres) («rose bleue » de Laval, grès de Normandie, charniers de Landéan?) (groupes GT3, GT5 et GT8)

- - des importations à l'échelle du territoire (grès et céramique glaçurée du Beauvaisis, céramiques de Saintonge, gobelets de Saint-Jean-de-la-Motte...) (groupes GT2, GT4, GT5)

- d'autres pour lesquelles il nous manque encore l'origine, comme le groupe 2-3. Le rapprochement effectué, dans un premier temps, avec les productions de Fontevrault ne semble pas probant (information orale de D. Prigent). Sur le site du Louvre, F. Ravoire indique également la présence d'un lot dont le descriptif semble comparable à nos fragments, et pour lequel elle ne livre pas de provenance, évoquant seulement des possibilités dans l'Ouest de la France (Maine, Vendée, Poitou ou Charente) ou en Champagne (Ravoire, Monnet, 1992, p. 41).

- enfin, bien qu'anecdotiques en nombre de fragments, les groupes 5-4 et 7 méritent d'être signalés comme témoins des réseaux commerciaux reconnus pour ces périodes avec l'Espagne (Hurst et al., 1986, p. 65-67) et la zone rhénane (ibid., p. 177-180).

Figure 19 : Carte des provenances des céramiques. Infographie : F. Labaune-Jean, Inrap. Figure 19: Map of the origins of ceramic.

101 Ces céramiques importées offrent des informations intéressantes sur les pratiques rennaises, même s'il faut les traiter prudemment en raison des quantités marginales qu'elles représentent dans les deux ensembles. La prédominance des marchés locaux pour répondre aux usages du quotidien, est incontestable ; les céramiques non locales servant de complément principalement par des vases intervenant comme contenant pour le transport et la diffusion des produits. Ainsi, les vases en grès normands et en « rose bleue » de Laval permettent le transport et le stockage du beurre breton. Leur essor est, en effet, directement lié à leurs qualités de légèreté, de dureté et d'imperméabilité, tout à fait adaptées au conditionnement du beurre. Enfin, la présence de la céramique de Laval à Rennes permet d'élargir la carte de diffusion de cette production auparavant reconnue jusqu'à Vitré (Naveau, 1998, p. 147).

\section{Les données morphologiques}

102 Faute d'autres études précises sur les formes céramiques locales, il est actuellement difficile d'établir une chronologie relative au sein des deux ensembles (tab. 2). Pour les 
latrines 1 (Us 2512, 2540 et 2702), l'essentiel du matériel céramique renferme des récipients en céramique commune locale. Les coquemars à lèvre haute rainurée (pot 3-1a et b), majoritaires, sont associés à des coquemars à embouchure verticale délimitée par une petite lèvre en bourrelet carré (pot 3-1c). Le vaisselier est complété par des tasses polylobées (tasse 1), des jattes (à lèvre rentrante ou en méplat éversé à l'horizontal, plat 2-1 à 2-4), mais aussi des pichets à col mouluré et bec pincé (pichet 3), des couvercles et des chauffe-plats, ainsi que des fragments de charniers à pâte grossière. Certains de ces vases possèdent une glaçure interne ou externe de teinte verte plus ou moins dense. Les importations sont représentées par des coupes en grès $\mathrm{du}$ Beauvaisis (GT 5-3) (Morisson, 1970, p. 43-72) et pots de Normandie (GT 5-1 et 5-2) (Flambart-Héricher, 2002 ; Dufournier et Flambart-Héricher, 1987), de la céramique de Laval (GT 3) (Naveau, 2001, p. 47-77) et des gobelets à pâte fine en provenance des ateliers de Saint-Jean-de-la-Motte (GT 4) (Husi, 2003, p. 30). En comparaison avec les données parisiennes (Ravoire, Monnet 1992, p. 40), on peut envisager que les coupes en grès du Beauvaisis aient pu être achetées pour les malades, de même que les gobelets à parois fines ; les deux autres groupes techniques (GT5-1 et GT3) représentant celui des contenants (cf. supra).

Tableau 2 : Répartition des individus par type de forme et par groupe technique. Table 2: Distribution of pottery by type of shape and by technical group.

\begin{tabular}{|c|c|c|c|c|c|}
\hline $\begin{array}{l}\text { Groupes } \\
\text { techniques }\end{array}$ & $\begin{array}{l}\text { Service de } \\
\text { table }\end{array}$ & $\begin{array}{l}\text { Préparation } \\
\text { Cuisson }\end{array}$ & $\begin{array}{l}\text { Service des } \\
\text { liquides }\end{array}$ & $\begin{array}{l}\text { Stockage } \\
\text { Transport }\end{array}$ & $\begin{array}{l}\text { Fonction indét. Ou } \\
\text { particulière }\end{array}$ \\
\hline GT 1 & $\begin{array}{l}\text { Chauffe- } \\
\text { plats } 1 \text { à } 3 \\
\text { Coupe 1-1, } \\
1-2 \\
\text { Tasse 1-1 }\end{array}$ & $\begin{array}{l}\text { Pots 2-1 à 3-6 } \\
\text { Pot } 9 \\
\text { Marmite 1-1 } \\
\text { Plats-jattes } 2,3 \\
\text { Couvercles } 1 \text { à } \\
3\end{array}$ & $\begin{array}{l}\text { Pichets } 3 \text { à } 5 \\
\text { Pichet } 7-1 \\
\text { Cruches } 1 \text { et } \\
2 \\
\text { Bouteille 1-1 }\end{array}$ & $\begin{array}{l}\text { Couvercles } 1 \text { à } 3 \\
\text { Pot } 1-5 ?\end{array}$ & $\begin{array}{l}\text { Pot 3-8 } \\
\text { Pot 6-1 } \\
\text { Terrine 2-1 } \\
\text { Lampe 1? }\end{array}$ \\
\hline
\end{tabular}




\begin{tabular}{|c|c|c|c|c|c|}
\hline GT 2 & $\begin{array}{l}\text { Assiettes 1-1 } \\
\text { et 1-2 } \\
\text { Plat-jatte 1-1 } \\
\text { Coupe 3-2 et } \\
3-3 \\
\text { Tasse 2-2 } \\
\text { Galettière } \\
1-1 ?\end{array}$ & $\begin{array}{l}\text { Pots 3-7, 3-9 } \\
\text { Pots 4-1, 4-2 } \\
\text { Pot } 12 \\
\text { Poêle 2-1 } \\
\text { Lèchefrite 1-1 } \\
\text { Galettière 1-1? }\end{array}$ & $\begin{array}{l}\text { Pichets } 1 \text { et } \\
2 \\
\text { Pichet } 6\end{array}$ & $\begin{array}{l}\text { Gourdes } 1-1 \text { et } \\
1-2\end{array}$ & \\
\hline GT 3 & & & & Pots 5-1 à 5-3 & \\
\hline GT 4 & & & Gobelet 1-1 & & \\
\hline GT 5 & $\begin{array}{l}\text { Coupe } 2-1 \text { et } \\
2-2\end{array}$ & & Pichet 8-1 & $\begin{array}{l}\text { Pot 1-1 à 1-3 } \\
\text { Gourde 1-2 }\end{array}$ & \\
\hline GT 6 & & & & & Pot 7-1 \\
\hline GT 7 & & & & Amphore 1-1 & \\
\hline GT 8 & & & & $\begin{array}{l}\text { Vases-réserves } \\
1-1 \text { et } 2-1\end{array}$ & Terrine 1-1 \\
\hline
\end{tabular}

Pour le dépotoir D2, le catalogue montre la grande variété et l'homogénéité du registre. Si l'éventail des formes de récipients est assez large, en terme quantitatif, il faut souligner la forte concentration du matériel dans un nombre restreint de formes (tab. 3 et 4). La céramique de production locale (avec ou sans glaçure) est illustrée par le coquemar, récipient incontournable présentant différents profils (pot 3-1 à 3-11). Il est associé à d'autres types en quantité moins importante: des pichets, des marmites à deux anses et des jattes profondes. S'y ajoutent des formes moins fréquentes mais significatives de nouveaux usages: couvercles, pichets à goulot étroit, lèchefrites, tasses... Pour la même période chronologique, Ph. Husi remarque les mêmes associations au sein du mobilier issu du comblement de latrines sur le site de la place Sicard à Tours, en liaison avec un hôtel-Dieu. (Jouquand, 1996, p. 156 et suivantes ; Jouquand et al., 1999). 
Tableau 3 : Répartition des vases par formes principales et par structures. (Récipients fermés). Infographie : F. Labaune-Jean, Inrap.

Table 3: Distribution by main forms and by structures. (jugs, jars and micellaneous).
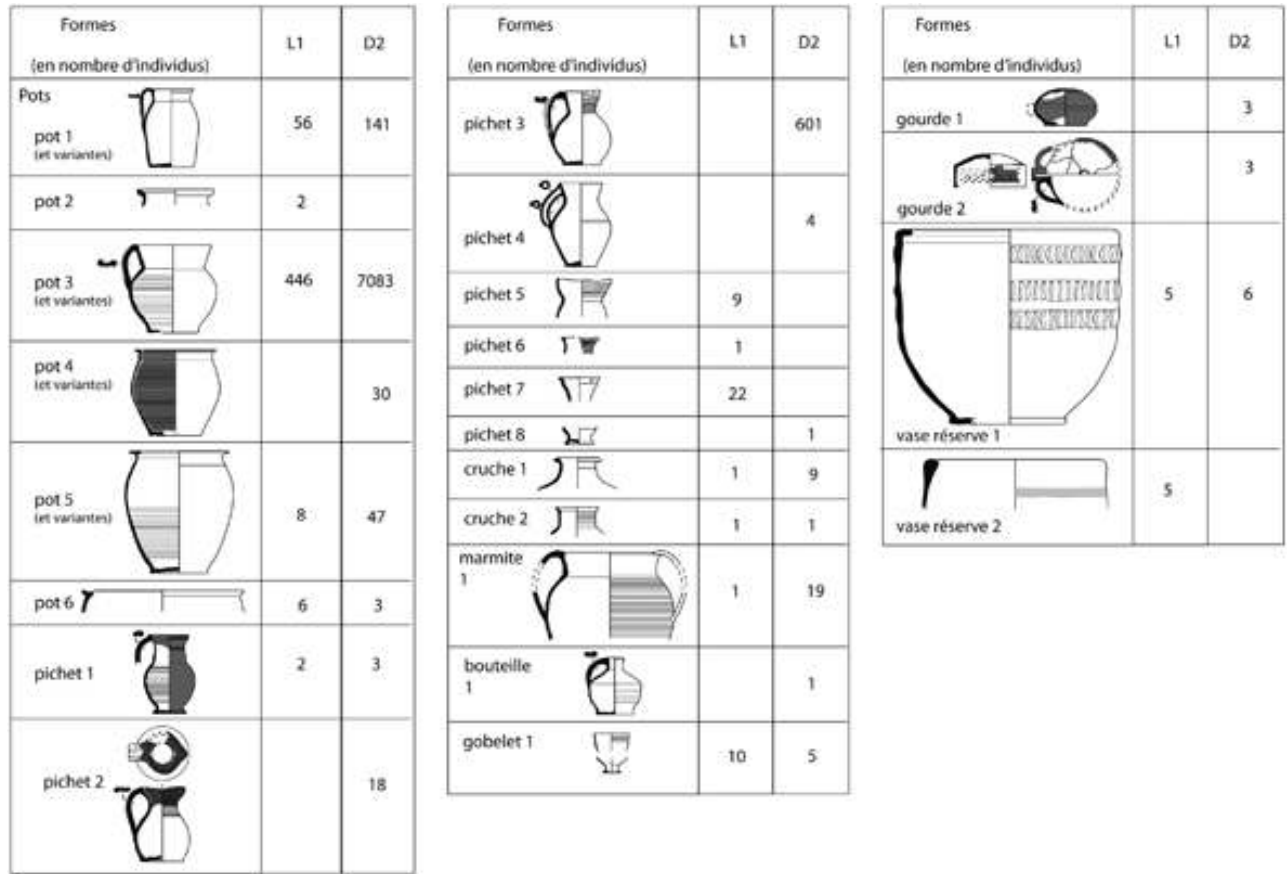

Tableau 4 : Répartition des vases par formes principales et par structures. (Récipients ouverts et ustensiles). Infographie : F. Labaune-Jean, Inrap.

Table 4: Distribution by main forms and by structures. (Dishes, bowls and micellaneous).
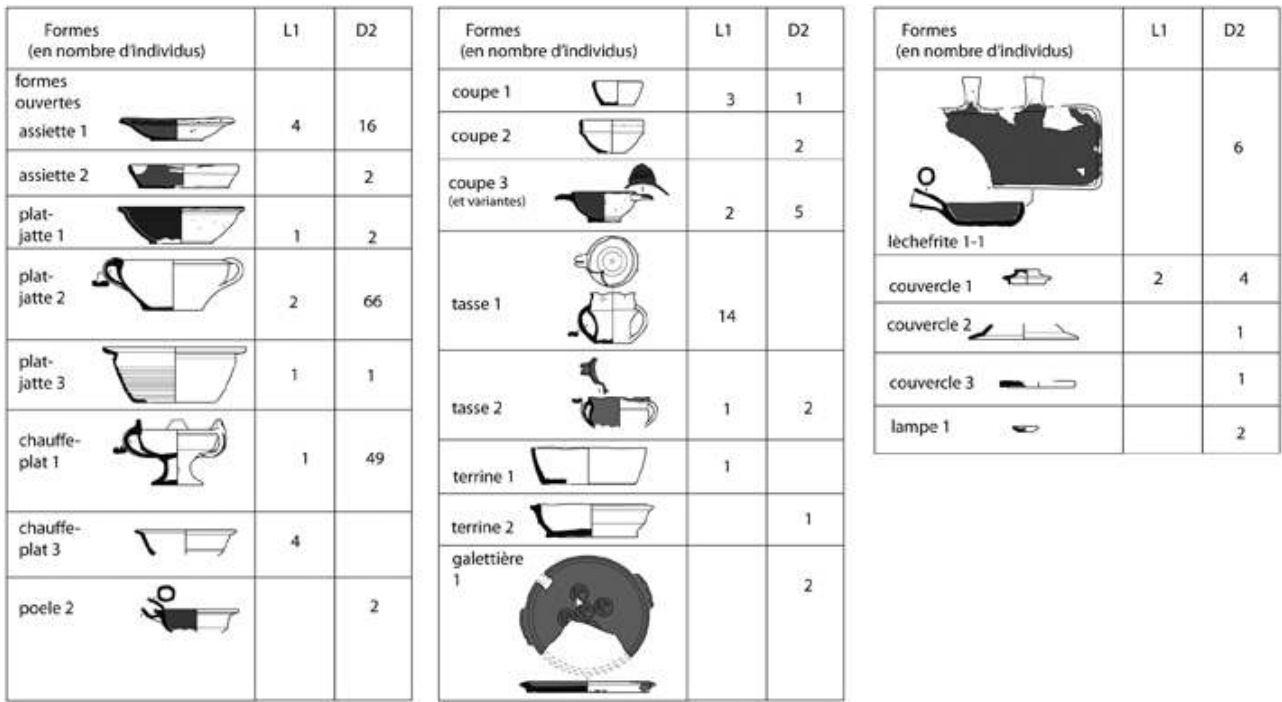

Les importations montrent des provenances diverses: Beauvaisis, Normandie, Saintonge, vallée du Rhin, région de la Sarthe (Saint-Jean-de-la-Motte et Laval) et Espagne (pour les amphores) (Blieck, 1985, p. 34-37). Le complément est assuré par de la vaisselle en bois (Dietrich, 2009) et de la verrerie (Labaune-Jean, 2010).

Les deux lots sont constitués en majorité de pots fermés (pots à cuire/coquemars et pichets) comme à Paris (Ravoire, Monnet, 1992, p. 38 ; fig. 18), correspondant aux récipients les plus fréquemment utilisés et illustrant ici les besoins de l'hôpital. Les fouilles du prieuré et de l'hôpital de St. Mary Spital à Londres montrent les mêmes 
phénomènes, tant pour la phase médiévale que la période moderne, puisque cet établissement fonctionne de $1050 \mathrm{au} \mathrm{XVIII}^{\mathrm{e}}$ siècle. (Thomas et al., 1997, p. 184-186). Le dépotoir D2, qui semble avoir été comblé rapidement au vu des données stratigraphiques observées sur le terrain, se trouvait sans doute à proximité de l'espace des cuisines si l'on se réfère aux nombreuses traces de cuisson laissées sur une bonne partie des récipients rejetés, même si le volume étudié et le manque de temps n'ont pas permis de quantifier cette remarque par l'estimation de la proportion de vases avec suie et effets de flammes par rapport aux vases sans traces. Il faut toutefois garder à l'esprit que cette répartition par usage reste indicative. Faute d'observations précises sur les récipients indiquant une utilisation particulière ou en l'absence d'analyses chimiques sur les résidus conservés dans certains vases, il demeure difficile de trancher pour un usage attitré d'une forme, si cet usage spécifique a existé. On ne peut pas négliger la notion d'opportunisme, l'usage d'un récipient pouvant être justifié par sa disponibilité à un moment donné précis. D. Alexandre-Bidon nous met ainsi en garde contre les travers des études céramologiques ayant tendance à cantonner les pots dans des usages précis (Alexandre-Bidon, 2005).

\section{Les informations particulières}

\section{Les contenances}

106 Le nombre important de vases intacts a permis d'aborder les contenances des pichets et des coquemars du dépotoir D2. Lors du premier tri, quatre volumes distincts et constants apparaissaient pour ces deux formes: un petit, deux moyens et un grand. Pour l'estimation des deux types, une limite arbitraire de mesure a été fixée à la base du col pour permettre les comparaisons. Vingt-cinq vases de chacune des deux formes ont été remplis jusqu'à cette limite. Le volume contenu mesuré, nous avons obtenu pour les coquemars (pot 3), quatre modules : 0,30 cl pour les petits, 1,25 1 pour les moyens, 21 pour les seconds moyens et 2,4 1 pour les grands. La contenance des pichets (type 3 ) donne : 0,50-0,60 cl pour les petits, 1,15/1,25 l pour les moyens, 1,60 l pour les seconds moyens et 2,1 1 pour les grands. L'étude en archives de Nicolas Cozic a permis de retrouver les unités de mesure en usage sur la ville pour la période. Les unités de mesure en usage figurent, en effet, dans des livres de compte de la ville et des frais de réception conservés aux archives municipales de Rennes pour la période $\mathrm{Xv}^{\mathrm{e}}$ $\mathrm{XVI}^{\mathrm{e}}$ siècles, ainsi dans le tableau de conversion des mesures anciennes de 1790 (publié par H. Sée) pour le département d'Ille-et-Vilaine (See, 1930). L'unité de mesure de base la plus utilisée est le pot. Appliquée aux vases du dépotoir D2, elle semble correspondre au troisième volume des pichets comme des coquemars. Les petits pichets correspondent à la chopine, et les seconds sont assez proches de la pinte. Les grandes contenances (estamal - près de 31 et quarte -près de $4 \mathrm{l}$ ) ne semblent pas représentées. Il faut certainement voir dans ces correspondances, la réalisation et l'emploi d'une production assez standardisée adaptée aux unités de mesure en usage (fig. 20).

Figure 20 : Correspondance des cinq contenances de pots et de pichets avec les dénominations des mesures en usage à Rennes à l'époque moderne. Infographie : F. Labaune-Jean, Inrap. Figure 20: Correspondence of five volumes of jars and jugs with the namings of the used measures in Rennes in the modern time. 


\section{Les vases déformés (fig. 21)}

Un nombre important a été recensé de vases intacts ou entiers présentant une déformation suffisamment importante pour permettre de les classer parmi les rebuts de cuisson, soit 58 vases : 5 pichets et 53 coquemars (37 pots 3-1 et 16 pots 3-5 et 3-6). Rencontrés normalement en contexte de production, dans les rejets d'ateliers, on peut donc s'interroger sur leur présence dans le comblement du dépotoir 2 . Une des solutions émises grâce aux archives est liée à la présence d'un marché aux céramiques très proche, situé sur la place Saint-Michel où les artisans de Chartres-de-Bretagne venaient écouler leurs productions (Beuchet, 1994). On peut alors envisager un approvisionnement de l'hôpital Sainte-Anne à plus faible coût à base des pots déformés mais malgré tout utilisables, surtout si l'on considère la forte demande nécessaire aux malades et la faible durée de vie des récipients. Ce phénomène tient donc peut-être à des accords particuliers passés entre les potiers et les hôpitaux, qui restent à démontrer par les données d'archives...

Figure 21 : Exemple de coquemars déformés à la cuisson. Cliché H. Paitier, Inrap.

Figure 21: Example of coquemars deformed in the cooking.

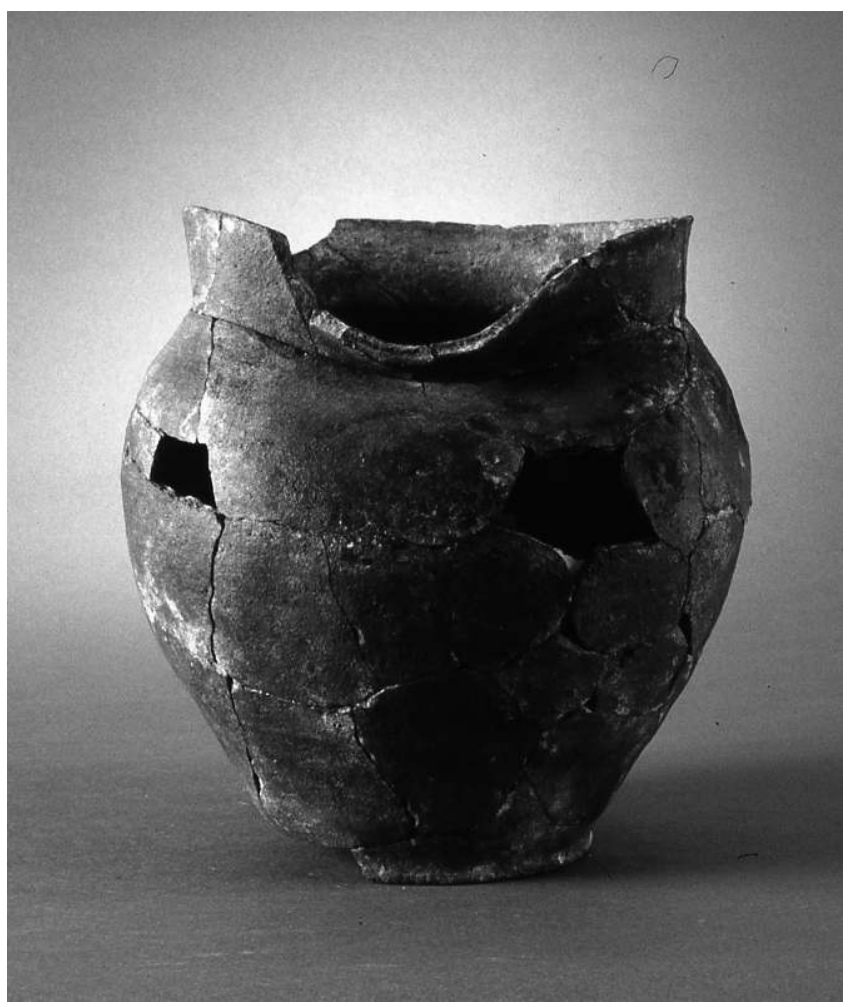

\section{Les récipients d'après les données d'archives}

En dehors de trois liasses (archives n 327 à 329 des archives municipales de Rennes ) consacrées à la gestion de biens immobiliers dépendants de l'établissement, les archives municipales de Rennes et départementales d'Ille-et-Vilaine présentent un fond relativement pauvre en données sur l'hôpital Sainte-Anne. Cependant, l'étude a pu être menée en partie grâce aux archives liées à l'hôpital Saint-Yves, auquel l'hôpital SainteAnne est associé dans la seconde moitié $\mathrm{du} \mathrm{xVI}^{\mathrm{e}}$ siècle. Le travail de dépouillement de $\mathrm{N}$. Cozic, orienté en fonction des questionnements liés aux découvertes de terrain a 
montré que si l'aménagement des latrines L1 apparaît dans les données d'agrandissement de l'établissement de soin, il n'existe aucune trace de la construction des maçonneries délimitant le dépotoir D2. D'une capacité d'accueil estimée entre 30 et 50 personnes logées (Cozic, 1998-2000), l'hôpital assure également le gîte et l'aumône de repas auprès des gens de passage (pèlerins, vagabonds, pauvres...; toutes les catégories sociales sont présentes dans l'établissement, les pratiques de l'époque étant d'y accueillir les veufs et veuves en échange d'une participation financière ou œuvrée), actions pour lesquelles il reçoit des aides particulières de la communauté de ville (Cozic, 1998-2000, p. 25. arch. mun. Rennes, liasse 113, quittance du 23 juillet 1497). À partir de 1498, il accueille les malades de l'épidémie de petite vérole, puis en 1507-1508 et 1550 , ceux de la peste, en raison de son emplacement dans les faubourgs de la ville. Ces affectations spécifiques et son éloignement des points d'approvisionnement en eau courante sont peut-être liés à l'abandon de l'établissement. En 1557, un acte mentionne l'union des deux établissements le 21 octobre (arch. mun. Rennes, liasse 327). Et à partir de 1566, un autre document en date du 24 novembre mentionne le regroupement des pensionnaires dans les nouveaux locaux de l'hôpital Saint-Yves pour améliorer la gestion (arch. mun. Rennes, liasse 317 ; Cozic, 1998-2000, p. 46). Si les livres de comptes (arch. mun. Rennes, liasse 1016, compte des prévôts de Saint-Yves de décembre 1583 à décembre 1584 et Arch. Dép. Ille-et-Vilaine, $H$ dépôt Rennes, art. $n^{\circ} 453$, compte du gardien de Sainte-Anne pour l'année 1561 ; Cozic, 1998-2000, p. 41-42) mentionnent les diverses denrées achetées par l'hôpital pour nourrir ses pensionnaires, ils indiquent rarement les ustensiles nécessaires à la mise en œuvre des repas. Seuls les pichets ("bues en terre ») mis à disposition dans les chambres et les salles, sont indiqués comme faisant l'objet d'un nettoyage régulier (Arch. Mun. Rennes, liasse 327 ; Cozic, 1998-2000, p. 44). On apprend aussi qu'au début du XVII ${ }^{\mathrm{e}}$ siècle, l'hôpital Saint-Yves revend un lot de poteries achetées pour leur contenu (beurre) (Arch. Mun. Rennes, liasse 1016, compte des prévôts de Saint-Yves 1624, fo 18 ; Cozic, 1998-2000, p. 44). Ce type de document introduit donc une notion nouvelle : le mobilier recueilli, tant dans les latrines que dans le dépotoir, ne reflète qu'une partie du vaisselier, le reste ayant pu être remis en service. Dans le cas particulier du dépotoir D2, il convient de s'interroger une nouvelle fois sur le rejet de récipients intacts et sans défauts apparents. Les hypothèses que l'on peut avancer, pour le moment, se rapportent soit à l'affectation de l'hôpital dans le traitement des pestiférés de l'épidémie de 1550, et donc, au rejet des récipients utilisés, si tant est que la notion sanitaire de l'époque aille jusque-là. Même si les mesures de prophylaxie ne sont pas encore véritablement organisées, les observations des médecins (notamment ceux du groupe des "contagionistes", partisans de la transmission par contact) conduisent à des mesures de pratiques ordinaires, tel que l'isolement des malades et l'évacuation des objets utilisés dans leur traitement (Audoin-Rouzeau, 2003, p. 223). L'absence de traces d'usage sur une partie d'entre eux peut aussi être liée à un usage "détourné » des récipients comme pot de chambre (pots 3) ou bassin de toilette (plat-jatte 2) par exemple, comme on peut le voir dans l'iconographie médiévale (Alexandre-Bidon, 2011). Il faut mentionner également la mauvaise gestion du gardien de Sainte-Anne, dénoncée dans un acte de 1557, pouvant peut-être expliquer la cause de ce "gaspillage» de récipients. (arch. mun. Rennes, liasse 327, actes d'union des hôpitaux Saint-Yves et Sainte-Anne, 21 octobre 1557 ; Cozic, 1998-2000, p. 44).

109 Les documents nous livrent également des informations sur les commandes de denrées alimentaires (Cozic, 1998-2000, p. 41) dont la présence a pu être confirmée ensuite à la 
fouille, par les analyses des restes palynologiques, entomologiques et fauniques (Clavel, 1998-2000 ; Clavel 2010), avec une alimentation carnée riche et variée témoignant de la volonté de répondre aux différents besoins des malades, les théories médicales en vigueur plaçant la nourriture au premier plan des soins, comme alicament (AlexandreBidon, 2008 ; Taillevent, 2001).

\section{La chronologie}

En plus des données d'archives, permettant de suivre la chronologie de l'établissement de sa fondation à son abandon au profit de l'hôpital Saint-Yves (Cozic, 1998-2000), les deux contextes ont livré du mobilier associé fournissant des données chronologiques complémentaires. Pour les latrines L1, une monnaie frappée entre 1500 et 1550 confirme les données de terrain, avec le remblaiement d'un contexte de la première moitié du $\mathrm{XVI}^{\mathrm{e}}$ siècle, à l'aide de terres renfermant du mobilier de la seconde moitié du $\mathrm{XV}^{\mathrm{e}}$ siècle. La datation du dépotoir principal (D2) est complétée par un mobilier très important. Les 25 monnaies du puisard donnent le terminus de 1553, avec des monnaies d'Henri II, ainsi qu'un jeton du milieu du Xvi ${ }^{\mathrm{e}}$ siècle (Pilet-Lemière, 1998-2000, inédit). $\mathrm{V}$. Montembault propose la période du premier tiers $\mathrm{du} \mathrm{XVI}^{\mathrm{e}}$ siècle pour les objets en cuir (Montembault, Bell 1998-2000)5. En dendrochronologie, V. Bernard (Bernard, 1998-2000, inédit) obtient des dates d'abattage comprises entre 1467 et 1489. Les formes de objets façonnés dans ces bois, tout comme les techniques de tissage des fragments de tissus correspondent également au XVI ${ }^{\mathrm{e}}$ siècle (Dietrich, 1998-2000; Dietrich, 2009; Cardon, 1998-2000, inédit). Les nefs gravées sur les dalles de schiste correspondent à des navires de la fin du Xve siècle (Carpentier, 1998-2000, inédit). La même datation peut être appliquée au style et à l'iconographie des enseignes de pèlerinage (Labaune, 1998-2000, inédit). Malgré une forte détérioration du matériau, les éléments de récipients en verre correspondent également à des récipients en usage dans cette même période (verres à boire à fond en piédouche ou à pied refoulé) (Labaune-Jean, 2010). Tous ces éléments associés à la céramique permettent donc d'avancer une date fiable pour l'utilisation du puisard comme dépotoir, dans le second quart du XVI ${ }^{\mathrm{e}}$ siècle avec le terminus vers le milieu du $\mathrm{XVI}^{\mathrm{e}}$ siècle.

\section{Conclusion}

111 L'étude de ces lots apporte des données essentielles pour les connaissances locales. En effet, à la différence d'autres régions comme à Tours (Husi, 1996) ou Paris (Ravoire, 1991 et 2006) où l'intérêt porté à cette céramique est plus ancien et plus riche en découvertes, le cas rennais ne comporte aucun référentiel et très peu de découvertes, à l'exception d'éléments isolés, souvent sans contexte. La variété et la complémentarité dans les formes recueillies ici permettent d'avoir un échantillonnage assez exhaustif de la vaisselle en usage pour une période bien définie (si l'on prend également en compte les lots antérieurs et postérieurs également issus de ce même site, Labaune-Jean 2009), avec cependant la réserve du contexte particulier. Pour certains types, une spécificité fonctionnelle semble indissociable du type de pâte utilisée. Cette première approche reste cependant lacunaire sur les différents groupes techniques rencontrés. Il faudrait envisager une analyse des textures pour voir s'il est possible de déterminer la nature des argiles et la provenance des productions de façon plus sûre. 
112 Il faut encore signaler les indices importants sur le type d'alimentation en usage, à mettre en liaison avec les récipients découverts, les fouilles en contextes hospitaliers médiévaux et modernes étant jusqu'à présent réduites à de rares interventions. Faute de découvertes comparables, il est en effet difficile d'établir pour le moment les spécificités réelles en contexte hospitalier, par rapport aux milieux d'habitats traditionnels. On notera, ainsi, une intervention à proximité de l'emplacement présumé de l'hôpital du Pas à Rodez (Catalo et al., 1995), la fouille de l'hôpital gantois à Lille (Fosse et al., 2003 ; Oueslati, 2006 ; Gubbellini et Boniface, 2002) ou encore l'hôtel-Dieu de Paris (Jehanno, 2004). S'ajoute enfin la notion de renouvellement fréquent de la vaisselle qui explique, en grande partie, l'importance du lot. En plus de la fragilité du matériau et de notions évoquées ci-dessus, certaines études montrent que les récipients étaient souvent changés au profit de "pots neufs ", d'où la découverte de pots intacts et sans défaut (À la fortune du pot, 1990, p. 33).

113 Il convient également de garder à l'esprit l'aspect sélectif de ce comblement car, en l'absence de données spécifiques à l'organisation de la gestion de l'évacuation des déchets par l'établissement hospitalier, ces deux ensembles offrent une vision du vaisselier à un moment donné, sans qu'il soit possible d'en estimer les manques ou les disproportions. Comme pour l'hôpital Saint-Yves, un acte (compte des prévôts de SaintYves 1525-1526) fait mention de l'emploi d'un charretier à cette fonction (Cozic, 1998-2000, Arch. Dép. d'Ille-et-Vilaine, H dépôt de Rennes, art. n 542), il faut peut-être envisager une personne en charge de l'évacuation des déchets en dehors de l'enceinte de l'établissement.

114 Les comparaisons avec d'autres contextes urbains publiés montrent l'existence de phénomènes particuliers à la région et de formes locales, comme les galettières à décor de poinçons, ou encore les tasses polylobées, sans comparaison pour le moment. Il faut toutefois regretter la difficulté de trouver des contextes similaires en milieu hospitalier, peu d'établissements de ce type et de même période ayant fait l'objet d'interventions archéologiques et encore moins de publications. Si les actes du colloque international d'Amiens en 2002 mentionnent la place et les relations des hôpitaux dans leur espace, il n'y ait pas fait référence au fonctionnement technique, à l'approvisionnement et aux pratiques de commerce (Racinet, 2002). On notera cependant des similitudes avec le mobilier issu des latrines $\mathrm{L} 3 \mathrm{du}$ site de la place Picard à Tours (Jouquand, 1996), tant pour les céramiques que pour les autres matériaux (bois, verre, cuir...). Par conséquent, il nous est difficile d'établir les spécificités du vaisselier, en dehors de la part prépondérante de la vaisselle d'usage courant. Les mêmes remarques ont été signalées pour les céramiques de l'hôpital St. Mary Spital de Londres, avec par contre, une prépondérance plus marquée des récipients destinés aux liquides (Thomas et al., 1997). Des décalages de datation existent avec d'autres villes, mais peuvent s'expliquer par le temps de diffusion et de copie des modèles en provenance des autres régions. Ainsi, par exemple, les cruches du dépotoir D2 observées par Ph. Husi sont déjà en usage au XIV ${ }^{e}$ siècle sur Tours. Ce phénomène avait déjà été évoqué par L. Beuchet pour l'époque médiévale (Beuchet, 1994, p. 68).

115 Grâce à cette fouille, nous avons pu acquérir une meilleure vision de la vie quotidienne des Rennais au début de l'époque moderne, pour des milieux relativement modestes en contexte hospitalier, même s'il reste encore des questions sans réponse, en attente de lots similaires en contexte d'habitat pour établir des comparaisons de typologie, d'approvisionnement, etc. 
116 Ce travail d'études doit beaucoup à la collaboration amicale des plusieurs personnes que nous tenons à remercier : Catherine Bizien-Jaglin, Denis Bruna, Daniel Dufournier, Anne-Marie Flambard-Héricher, Anne-Marie Fourteau, Philippe Husi, Anne-Marie Jouquand, Jacques Naveau, Maria Teresa Penna, Daniel Prigent, Fabienne Ravoire.

\section{BIBLIOGRAPHIE}

ALEXANDRE-BIDON D., 2005 - Une archéologie du goût. Céramique et consommation, Paris, Picard collection «Espaces médiévaux », 301 p.

ALEXANDRE-BIDON D., 2008 - « La santé au Moyen Âge », Catalogue d'exposition de la Tour Jean Sans Peur, Paris, $39 \mathrm{p}$.

ALEXANDRE-BIDON D., 2011 - « Au lit au Moyen Âge », Catalogue d'exposition de la Tour Jean Sans Peur, Paris, $45 \mathrm{p}$.

AUDOIN-ROUZEAU F., 2003 - Les chemins de la peste. Le rat, la puce et l'homme, Rennes, Presses universitaires de Rennes, coll. Histoire, $371 \mathrm{p}$.

BERNARD V., 1998-2000 - « Les bois du site de la place Sainte-Anne », in POUILLE D., 1998-2000, Rennes. Fouilles du métro VAL. Station Place Sainte-Anne, Document final de synthèse de fouille préventive, Rennes, AFAN-SRA Bretagne, Tome 6-2.

BEUCHET L., 1994 - « La potterye de Fontenay. L'artisanat céramique à Chartres-de-Bretagne à la fin du Moyen Âge ", Mémoire de maîtrise d'Histoire de l'Art et d'Archéologie, université de Rennes II. (tapuscrit).

BEUCHET L., DUFOURNIER D. et FICHET DE CLAIRFONTAINE F., 1990 - « Les ateliers de potiers médiévaux de Fontenay à Chartres-de-Bretagne (Ille-et-Vilaine) du XI ${ }^{\mathrm{e}}$ au XIV ${ }^{\mathrm{e}}$ siècle », Archéologie médiévale, 28, Paris, p. 71-120.

BEUCHET L., LABAUNE F., PICAULT CH. et al., 2004 - « Trois lots de mobilier du XVI ${ }^{\mathrm{e}}$ siècle provenant du château du Guildo (Côtes-d'Armor) ", Revue archéologique de l'Ouest, 21, Rennes, p. 189-223.

BLIECK G., 1985 - «Céramiques espagnoles du bas Moyen Âge à Lille », Amphora, 41, septembre 1985, p. 34-37.

BUCUR I., DUFOURNIER D., GOULPEAU L. et al., 1984 - « La céramique à œil de perdrix et la production de La Hardelière à Laval », Archéologie médiévale, tome XIV, p. 171 à 219.

CARDON D., 1998-2000 - « Les textiles du dépotoir de l'hôpital », in POUILLE D., 1998-2000, Rennes.

Fouilles du métro VAL. Station Place Sainte-Anne, Document final de synthèse de fouille préventive, Rennes, Afan-SRA Bretagne, tome 6-1.

CARPENTIER V., 1998-2000 - « Les graffitis de bateaux du dépotoir de l'hôpital », in POUILLE D., 1998-2000, Rennes. Fouilles du métro VAL. Station Place Sainte-Anne, Document final de synthèse de fouille préventive, Rennes, Afan-SRA Bretagne, Tome 6-1.

CHAPELOT J., 1975-1976 - Potiers de Saintonge. Huit siècles d'artisanat rural, Catalogue d'exposition, Paris, $127 \mathrm{p}$. 
CLAVEL B., 2010 - « Données archéozoologiques et fouilles d'hôpitaux : l'hôtel-Dieu de Rennes », in LE CLECH-CHARTON S. (dir.), Les établissements hospitaliers en France du Moyen Âge au XIX siècle, Actes du colloque Espaces, objets, populations dans les établissements hospitaliers du Moyen Âge au XIX ${ }^{\mathrm{e}}$ siècle, Tonnerre 4 et 5 septembre 2008, Dijon, Éditions universitaires, p. 365-379.

COZIC N., 1998-2000 - «Étude d'archives », in POUILLE D., 1998-2000, Rennes. Fouilles du métro VAL. Station Place Sainte-Anne, Document final de synthèse de fouille préventive, Rennes, Afan-SRA Bretagne, tome 5.

DIETRICH A., 1998-2000 - « Les objets en bois du site de la place Sainte-Anne », in POUILLE D., 1998-2000, Rennes. Fouilles du métro VAL. Station Place Sainte-Anne, Document final de synthèse de fouille préventive, Rennes, Afan-SRA Bretagne, tome 6-1. (Inédit).

DIETRICH A., 2009 - «Les petits métiers du bois et les arts de la table à la fin du Moyen Âge », in DIETRICH A. et RAVOIRE F. (dir), 2009, La cuisine et la table dans la France de la fin du Moyen Âge, Actes du colloque de Sens (Janvier 2004), Caen : publication du Crahm, $450 \mathrm{p}$.

DUBANT D., 2003 - « Les enceintes de Rennes », in « La Bretagne ducale », Histoire médiévale nº 3, hors-série, août-octobre 2003, p. 48-53.

DUFOURNIER D. et FLAMBARD-HERICHER A.-M., 1987 - « Réflexions à propos de l'apparition du grès en Europe occidentale ", in La céramique ( $v^{e}$-XIXe siècles). Fabrication. Commercialisation. Utilisation, Actes du $1^{\text {er }}$ congrès international d'archéologie médiévale de Paris, 1985, Caen, p. 139-147.

FAURE-BOUCHARLAT E. et LEYGE F., 1990 - À la fortune du pot. La cuisine et la table à Lyon et à Vienne $X^{e}$ XIX ${ }^{e}$ siècles d'après les fouilles archéologiques, Catalogue d'exposition, Lyon, $234 \mathrm{p}$.

FICHET DE CLAIRFONTAINE F. (dir.), 1996 - « Ateliers de potiers médiévaux en Bretagne », Dossiers d'Archéologie française, $\mathrm{n}^{\circ}$ 55, Paris, éd. Maison des sciences de l'Homme, 165 p.

FLAMBARD-HERICHER A.-M., 2002 - Potiers et poteries du Bessin. Histoire et archéologie d'un artisanat rural $d u X^{e}$ au XXe siècle, en Normandie, Caen, publications du CRAHM, $407 \mathrm{p}$.

FOSSE G., DELFOLIE K., REVILLION S. et ROUMEGOUX Y., 2003 - « Un hôpital fondé au XVe siècle : l'hôpital Gantois ", Archéologie en Nord-Pas-de-Calais, $\mathrm{n}^{\circ}$ 7, Lille, SRA Nord-Pas-de-Calais.

GOUPIL F. et COZIC N., 1991 - Projet VAL, Rapport de diagnostic préliminaire, Rennes, SRA Bretagne. GUILLIEUX J., 1981 - « Fouille de sauvetage sur le site « la Chausse-Paillere » en Saint-Jean-de-laMotte », Bulletin de la société d'émulation de la Sarthe, $n^{\circ}$ 558, Laval, p. 41-60.

GUBELLINI L. et BONIFACE F., 2002 - « Céramique et verrerie en milieu hospitalier au XVI ${ }^{\mathrm{e}}$ siècle : l'hospice Gantois à Lille », Revue du Nord, tome 84, Lille, p. 145-169.

HURST J.G., NEAL D.S. et VAN BEUNINGEN H.J.E., 1986 - « Pottery produced and traded in North-West Europe 1350-1650», Rotterdam Papers VI, Rotterdam, 281 p.

HUSI PH., 1996 - «Périodisation et essai de détermination des céramiques locales et importées utilisées à Tours du XIV ${ }^{\mathrm{e}}$ au XVII ${ }^{\mathrm{e}}$ siècle à partir des fouilles récentes ", Archéologie médiévale 28, Paris, p. 145-199.

HUSI PH. (dir.), 2003 - « La céramique médiévale et moderne du Centre-Ouest de la France (XI ${ }^{\mathrm{e}}$-XVIII siècle). Chrono-typologie de la céramique et approvisionnement de la vallée de la Loire moyenne », 20e supplément à la Revue Archéologique du Centre de la France, Tours, $110 \mathrm{p}$.

HUSI PH., 2011 - « Réseau d'information sur la céramique médiévale et moderne (Iceramm) : présentation et premier bilan », in BOCQUET-LIENARD A., et FAJAL B. (dir.), 2011, À propos de l'usage, de la 
production et de la circulation des terres cuites dans l'Europe du Nord-Ouest autour des XIVe-XVI $I^{e}$ siècles, Actes des tables rondes du CRAHM, 7-8 décembre 2007), CRAHM, Caen, p. 25-30.

JEHANNO CH., 2004 - « Un hôpital au cœur de la ville : inscription spatiale et insertion sociale de l'hôtel-Dieu de Paris au Moyen Âge », in DE SPIEGELER P. (dir.), 2004, « Hôpitaux et maladreries au Moyen Âge : espace et environnement, (Actes du colloque international d'Amiens-Beauvais, 22-24 novembre 2002) », Histoire médiévale et Archéologie, tome 17, CAHMER, Amiens, p. 101-112.

JOUQUAND A.-M., 1996 - Tours, Place François Picard, DFS de sauvetage programmé, Orléans, SRA Centre, 211 p. inédit).

JOUQUAND A.-M., CHAMPAGNE F., RODIER X., HUSI PH. et WITTMANN A., 1999 - « La fouille des abords de la cathédrale de Tours (Indre-et-Loire) : Antiquité - haut Moyen Âge », Revue archéologique du Centre de la France, vol. 38, Tours, p. 7-98.

LABAUNE F., 1998-2000 - « Les lots de mobilier d'époque moderne et médiévale », in POUILLE D., 1998-2000, Rennes. Fouilles du métro VAL. Station Place Sainte-Anne, document final de synthèse de fouille préventive, Rennes, Afan-SRA Bretagne, vol. 4-1 et 4-2.

LABAUNE-JEAN F., 2010 - « Le verre soufflé-moulé dans les contextes hospitaliers à Rennes, place Sainte-Anne ", in FONTAINE CH., 2010, D'Ennion au Val Saint-Lambert. Le verre soufflé-moulé, (actes des $23^{\mathrm{e}}$ rencontres de l'AFAV, Bruxelles-Namur 2008), Bruxelles, IRPA, Publication Scienta Artis no 5, p. 391-396.

LABAUNE-JEAN F., 2009 - « Le vaisselier rennais du XV au XVII ${ }^{\mathrm{e}}$ siècle ", in DIETRICH A. et RAVOIR F. (dir), 2009, La cuisine et la table dans la France de la fin du Moyen Âge, Actes du congrès de Sens, janvier 2004, Caen : publication du Crahm, p. 411-424.

LABAUNE-JEAN F., 2010 - « Rennes, place Sainte-Anne. Aperçu du mobilier de l'hôpital », in LE CLECHCHARTON S. (dir.), 2010, Les établissements hospitaliers en France du Moyen Âge au XIXe siècle, Actes du colloque Espaces, objets, populations dans les établissements hospitaliers du Moyen Âge au XIX ${ }^{\mathrm{e}}$ siècle, Tonnerre 4 et 5 septembre 2008, Dijon : éditions universitaires, p. 255-266.

LABAUNE-JEAN F. et POUILLE D., 2011 - « Un dépotoir en contexte hospitalier à Rennes, place SainteAnne. Aperçu du vaisselier en usage (fin $\mathrm{Xv}^{\mathrm{e}}$-début XVI ${ }^{\mathrm{e}}$ siècle) ", in BOCQUET-LIENARD A. et FAJAL B. (dir.), 2011, À propos de l'usage, de la production et de la circulation des terres cuites dans l'Europe du Nord-Ouest (XIV ${ }^{e}-\mathrm{XVI}^{e}$ siècle, Actes de la table-ronde 7-8 décembre Caen, Caen, publications du Crahm, p. 231-244.

LEFEVRE N. et MEYER N., 1988 - « Les lampes en céramique des fouilles urbaines de Saint-Denis », Archéologie médiévale, tome XVIII. Paris, p. 73 à 111.

LE GOFF J., 1980, Histoire de la France urbaine, la ville médiévale, Paris, Seuil, 656 p.

MONTEMBAULT V. et BELL B., 1998-2000 - «Étude de la collection de cuir découverte sur le site de la place Sainte-Anne à Rennes ", in POUILLE D., 1998-2000, Rennes. Fouilles du métro VAL. Station Place Sainte-Anne, Document final de synthèse de fouille préventive), Rennes, Afan-SRA Bretagne, Tome 6-1.

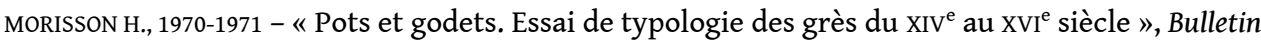
du groupe de recherches et d'études de la céramique du Beauvaisis, no 3, Beauvais, p. 43-72.

NAVEAU J., 1998 - Le chasseur, l'agriculteur et l'artisan. Guide du musée archéologique départemental de Jublains, Laval, 174 p.

NAVEAU J., 2001 - « Les potiers lavallois au début des temps modernes », La Mayenne : archéologie, histoire, $\mathrm{n}^{\circ}$ 24, Laval, p. 47-77. 
OUESTALI T., 2006 - «L'alimentation carnée en milieu hospitalier au XVI ${ }^{\mathrm{e}}$ siècle à partir des fouilles de l'hospice Gantois Lille (Nord) », Revue du Nord, tome 88, Lille, p. 69-78.

PILET-LEMIÈRE J., 1998-2000 - « Le lot monétaire de l'hôpital », in POUILLE D., 1998-2000, Rennes. Fouilles du métro VAL. Station Place Sainte-Anne, Document final de synthèse de fouille préventive, Rennes, Afan-SRA Bretagne, Tome 6-1.

POUILLE D., 1998-2000 - Rennes. Fouilles du métro VAL. Station Place Sainte-Anne, Document final de synthèse de fouille préventive, Rennes, Afan-SRA Bretagne, 6 volumes.

RACINET PH. (dir.), 2004 - « Hôpitaux et maladreries au Moyen Âge : espace et environnement », Histoire médiévale et archéologie, Actes du colloque international d'Amiens-Beauvais, 22-24 novembre 2002, volume 17, Amiens, CAHMER.

RAVOIRE F., 1991 - « Un ensemble céramique du XVI siècle : la fosse L1 des thermes de Cluny à Paris », Archéologie médiévale, 21, Paris, p. 209-270.

RAVOIRE F., 2006 - «Approvisionnement céramique et mode d'alimentation dans les communautés religieuses sous l'Ancien Régime. L'exemple de l'abbaye de Chelles (Seine-Maritime) et du couvent des Feuillantines à Paris ", in CLAVEL B. (dir.), 2006, Production alimentaire et lieux de consommation dans les établissements religieux au Moyen Âge et à l'époque moderne, Actes du colloque de Lille, 16, 17 et 18 octobre 2003, Histoire médiévale et archéologie, vol. 19-2006, Amiens, p. 301-325. RAVOIRE F. et MONNET C., 1992 - « La céramique médiévale », in MONNET C. (dir.), 1992, L'évacuation des déchets en milieu urbain au Bas Moyen Age. L'exemple des fosses à fond perdu de la Cour Napoléon du Louvre à Paris (XIII $-\mathrm{XV}^{e}$ siècles) et mesures diverses pour assainir les villes, Louvain la Neuve, $165 \mathrm{p}$. SEE H., 1930 - « De l'équivalence des anciennes et des nouvelles mesures dans le département d'Illeet-Vilaine ", Annales de Bretagne, vol. 39, Rennes, p. 242-250.

TAILLEVENT, 2001 - Le viandier d'après l'édition de 1486, Houilles, éd. Manucius, 73 p.

THOMAS CH., SLOANE B. et PHILLPOTTS CH., 1997 - « Excavations at the Priory and Hospital of St Mary Spital », London, MoLAS Monograph I, London, Museum of London, Archaeology Service, 267 p.

\section{NOTES}

1. Le dossier documentaire de l'hôpital Sainte-Anne souffre de nombreuses lacunes. Il faut en particulier mentionner l'absence d'un fonds d'archives cohérent. Cette absence tient au fait que dans la seconde moitié du $\mathrm{XVI}^{\mathrm{e}}$ siècle, l'hôpital Sainte-Anne a été rattaché à l'hôpital Saint-Yves, puis a cessé de fonctionner. De ce fait, ses archives propres n'ont pas été préservées. Pour aborder cette structure hospitalière, il faut donc étudier les fonds relatifs à Saint-Yves ou les comptes des miseurs de la ville de Rennes, fonds malheureusement incomplets qui recèlent un certain nombre d'informations éparses relatives à Sainte-Anne. Il convient également de signaler ici l'existence d'un fonds privé n'ayant pu être étudié, relatif à la famille Brûlon de la Musse, qui figure parmi les principaux bienfaiteurs de l'hôpital Sainte-Anne au début du XVI ${ }^{e}$ siècle. (propriétaire : M. Hervé, château du Val à Planguenoual, Côtes-d'Armor).

2. Coordonné par Ph. Husi, à l'université de Tours, ce groupe vise à mettre en place un système de classification similaire sur l'Europe du Nord-Ouest de manière à établir des outils des comparaisons régionales sur le vaisselier entre les $\mathrm{VI}^{\mathrm{e}}$ et $\mathrm{XVII}{ }^{\mathrm{e}}$ siècles. (site : http//iceramm.univ-tours.fr) (Husi, 2011). 


\section{RÉSUMÉS}

Cette présentation fait suite à un premier article publié dans les actes de la table ronde de Caen en 2007, où le lot du dépotoir D2 est évoqué dans son ensemble, montrant de façon succincte les formes et les groupes techniques associés, sans développer la typologie (Labaune, Pouille, 2011). Les lots découverts sur ce site hospitalier par leur volume exceptionnel, constituent des ensembles de premier ordre pour l'étude céramique de la cité médiévale et moderne et sont donc la base de la typo-chronologie présentée ici par type de récipient avec une classification des pâtes, de façon à établir les bases d'un référentiel rennais pour la période: fin $\mathrm{Xv}^{\mathrm{e}}$-première moitié du XVI ${ }^{\mathrm{e}}$ siècle.

This presentation follows upon a first article published in the acts of the round table of Caen in 2007 , when the set of the garbage dump D2 is evoked in general, showing in a brief way the forms and the technical groups, without developing the typology. Lots discovered on this hospital site by their exceptional volume, constitute excellent sets for the ceramic study of the medieval and modern city and they are the base of the typo-chronology presented here by type of bowl with a classification of fabrics, so as to establish the bases of Rennes reference table for this period : the end of the $15^{\text {th }}$ century-first half of the $16^{\text {th }}$ century.

\section{INDEX}

Keywords : ceramics, rubbish dump, medieval hospital, 15th century, 16th century, typology

\section{AUTEURS}

\section{FRANÇOISE LABAUNE-JEAN}

Assistante d'études, Inrap Grand-Ouest (Cesson-Sévigné), UMR 6566 CReAAH.

\section{DOMINIQUE POUILLE (AVEC LA COLLABORATION DE)}

Responsable d'opération, Inrap Grand-Ouest (Cesson-Sévigné), UMR 6566. 